\title{
ESSENTIAL KILLING FIELDS OF PARABOLIC GEOMETRIES
}

\author{
ANDREAS ČAP AND KARIN MELNICK
}

\begin{abstract}
We study vector fields generating a local flow by automorphisms of a parabolic geometry with higher order fixed points. We develop general tools extending the techniques of [1, 2], and [3], and we apply them to almost Grassmannian, almost quaternionic, and contact parabolic geometries, including CR structures. We obtain descriptions of the possible dynamics of such flows near the fixed point and strong restrictions on the curvature; in some cases, we can show vanishing of the curvature on a nonempty open set. Deriving consequences for a specific geometry entails evaluating purely algebraic and representation-theoretic criteria in the model homogeneous space.
\end{abstract}

Dedicated to Michael Eastwood on the occasion of his 60th birthday.

\section{INTRODUCTION}

An array of results in differential geometry tell us that geometric structures admitting a large group of automorphisms are special and must have a particularly simple form. For example, a Riemannian manifold $M^{n}$ with $\operatorname{Isom}(M)$ of maximum possible dimension $\frac{n(n+1)}{2}$ must have constant sectional curvature and thus be a space form. More generally, the maximal dimension for the Lie algebra of Killing vector fields on a

Date: October 18, 2018.

This project was initiated during the second author's Junior Research Fellowship at the Erwin Schrödinger Institute in Vienna, and was continued during a workshop at the ESI, "Cartan connections, geometry of homogeneous spaces, and dynamics." Cap is supported by Fonds zur Förderung der wissenschaftlichen Forschung, project P23244-N13, and Melnick is supported by NSF grant DMS-1007136. 
Riemannian manifold, or for the Lie algebra of infinitesimal automorphisms for many classical geometric structures, can be only attained on open subsets of a homogeneous model.

In some cases, the existence of a single automorphism or infinitesimal automorphism of special type restricts the geometry. Special automorphisms that exists for some geometric structures are those that equal the identity to first order at a point; note that because of the exponential map, Riemannian metrics never admit such automorphisms, except the identity. The projective transformations of projective space $\mathbf{R P}^{n}$, on the other hand, do include such automorphisms: there is Id $\neq g \in \operatorname{Aut}\left(\mathbf{R P}^{n}\right)$ with $g . x=x$ and $D g_{x}=$ Id. The space $\mathbf{R} \mathbf{P}^{n}$ viewed as a homogeneous space of the group of projective transformations is the model for classical projective structures. Such a structure on a manifold $M$ is an equivalence class $[\nabla]$ of torsion-free linear connections on $T M$ having the same sets of geodesics up to reparametrization. An automorphism is a diffeomorphism of $M$ preserving $[\nabla]$, or, equivalently, preserving the corresponding family of geodesic paths as unparametrized curves.

For $M$ connected, an automorphism of a classical projective structure on $M$ is uniquely determined by its two-jet at a single point. Non-trivial automorphisms fixing a point to first order are examples of essential automorphisms - ones not preserving any connection in the projective class $[\nabla]$. Nagano and Ochiai [1] proved that if a compact, connected manifold $M^{n}$ with a torsion-free connection admits a nontrivial vector field for which the flow is projective and trivial to first order at a point $x_{0}$, then $M$ is projectively flat on a neighborhood of $x_{0}$ - that is, locally projectively equivalent to $\mathbf{R} \mathbf{P}^{n}$.

Pseudo-Riemannian conformal structures may also admit non-trivial automorphisms which equal the identity to first order in a point. In this case, Frances and the second author prove analogous results in [2] and [3. Their theorems say that if a conformal vector field $X$ vanishes at a point $x$, and if the flow $\left\{\varphi_{X}^{t}\right\}_{t \in \mathbf{R}}$ is unbounded but has precompact differential at $x$, then the manifold is conformally flat on a nonempty open set $U$ with $x \in \bar{U}$. 
Both proofs make use of the Cartan geometry canonically associated to the structures in question and of the contracting - though not necessarily uniformly contracting-dynamics of the given flows. In both cases, the Cartan geometry is a parabolic geometry, one for which the homogeneous model is $G / P$ for $G$ a semisimple Lie group and $P$ a parabolic subgroup. An introduction to the general theory of parabolic geometries can be found in [4]. See [5] and [6] for general results on automorphisms and infinitesimal automorphisms.

In this article, we develop machinery to apply these ideas to study the behavior of a certain class of flows fixing a point that include the projective and conformal flows described above, in the general setting of parabolic geometries. Our results lead to descriptions of the possible dynamics of such flows near the fixed point and to strong restrictions on the curvature. In some cases, we can show vanishing of the curvature on a nonempty open set. Deriving consequences for a specific geometry entails evaluating purely algebraic and representation-theoretic criteria for the pair $(G, P)$.

\subsection{Background.}

1.1.1. Cartan geometries of parabolic type. Let $G$ be a semisimple Lie group with Lie algebra $\mathfrak{g}$. A parabolic subalgebra of $\mathfrak{g}$ can be specified by a $|k|$-grading for some positive integer $k$, which is a grading of $\mathfrak{g}$ of the form $\mathfrak{g}=\mathfrak{g}_{-k} \oplus \cdots \oplus \mathfrak{g}_{k}$ such that no simple ideal is contained in the subalgebra $\mathfrak{g}_{0}$, and such that the subalgebra $\mathfrak{g}_{-}=\oplus_{i<0} \mathfrak{g}_{i}$ is generated by $\mathfrak{g}_{-1}$. The parabolic subalgebra determined by the grading is then $\mathfrak{p}=\oplus_{i \geq 0} \mathfrak{g}_{i}$, and a parabolic subgroup $P<G$ is a subgroup with Lie algebra $\mathfrak{p}$. It is a fact that

$$
N_{G}^{0}(\mathfrak{p}) \leq P \leq N_{G}(\mathfrak{p})
$$

where $N_{G}(\mathfrak{p})$ is the normalizer and $N_{G}^{0}(\mathfrak{p})$ is its connected component of the identity. The center of $\mathfrak{g}_{0}$ contains the grading element $A$, for which each $\mathfrak{g}_{i}$ is an eigenspace of $\operatorname{ad}(A)$ with eigenvalue $i$. The $|k|-$ gradings of a given Lie algebra $\mathfrak{g}$ correspond to subsets of the simple roots when $\mathfrak{g}$ is complex, and subsets of simple restricted roots for $\mathfrak{g}$ 
real, associated to a choice of Cartan subalgebra (which is maximally non-compact in the real case); see section 3.2 of [4].

Defining $\mathfrak{g}^{i}=\oplus_{j \geq i} \mathfrak{g}_{j}$ makes $\mathfrak{g}$ into a filtered Lie algebra such that $\mathfrak{p}=\mathfrak{g}^{0}$. The parabolic subgroup $P$ acts by filtration-preserving automorphisms under the adjoint action. The subgroup $G_{0}<P$ preserving the grading of $\mathfrak{g}$ has Lie algebra $\mathfrak{g}_{0}$. Denote $\mathfrak{p}_{+}=\mathfrak{g}^{1}$, and let $P_{+}<P$ be the corresponding subgroup; it is unipotent and normal in $P$, and $\exp : \mathfrak{p}_{+} \rightarrow P_{+}$is a diffeomorphism. Then $G_{0} \cong P / P_{+}$, and it is closed and reductive.

Definition 1.1. Let $G$ be a Lie group with Lie algebra $\mathfrak{g}$ and $P$ a closed subgroup. A Cartan geometry on $M$ modeled on the pair $(\mathfrak{g}, P)$ is a triple $(M, B, \omega)$, where

(1) $\pi: B \rightarrow M$ is a principal P-bundle

(2) $\omega \in \Omega^{1}(B, \mathfrak{g})$ is the Cartan connection, satisfying

(a) for all $b \in B$, the restriction $\omega_{b}: T_{b} B \rightarrow \mathfrak{g}$ is a linear isomorphism

(b) for all $p \in P$, the pullback $R_{p}^{*} \omega=A d\left(p^{-1}\right) \circ \omega$

(c) for all $X \in \mathfrak{p}$, if $\tilde{X}$ is the fundamental vector field $\tilde{X}(b)=$ $\left.\frac{\mathrm{d}}{\mathrm{d} t}\right|_{0} b \cdot e^{t X}$, then $\omega(\tilde{X})=X$.

The Cartan connection generalizes the left-invariant Maurer-Cartan form $\omega_{G}$ on $G$. The following curvature is a complete obstruction to local isomorphism of $(M, B, \omega)$ to the homogeneous model $\left(G / P, G, \omega_{G}\right)$.

Definition 1.2. The curvature of the Cartan connection $\omega$ is the twoform $K \in \Omega^{2}(B, \mathfrak{g})$ given by

$$
K(\xi, \eta)=d \omega(\xi, \eta)+[\omega(\xi), \omega(\eta)]
$$

Definition 1.3. A parabolic geometry on a manifold $M$ is a Cartan geometry on $M$ modeled on $(\mathfrak{g}, P)$ for $G$ a semisimple Lie group and $P$ a parabolic subgroup.

The Cartan connection $\omega$ gives rise to natural local charts on $B$ as follows. To each $A \in \mathfrak{g}$ corresponds an $\omega$-constant vector field $\tilde{A} \in$ $\mathfrak{X}(B)$, characterized by $\omega(\tilde{A}) \equiv A$. Note that $\tilde{A}$ is the fundamental 
vector field if $A \in \mathfrak{p}$. For any $b \in B$, and sufficiently small $A \in \mathfrak{g}$, define $\exp (b, A)=\exp _{b}(A)$ to be the image of $b$ under the time-one flow along $\tilde{A}$. There is a neighborhood $U$ of $0 \in \mathfrak{g}$ on which $\exp _{b}$ is defined and a diffeomorphism onto an open subset of $B$. Composing the projection $\pi$ with the restriction of $\exp _{b}$ to any linear subspace in $\mathfrak{g}$ complementary to $\mathfrak{p}$, we obtain a local chart on $M$. The exponential map gives rise to a notion of distinguished curves and to normal coordinates on a parabolic geometry:

Definition 1.4. Consider a parabolic geometry on $M$ of type $(\mathfrak{g}, P)$.

- For $X \in \mathfrak{g}$, an exponential curve in $M$ is the projection to $M$ of a curve $t \mapsto \exp (b, t X)$ for some $b \in B$. It is a distinguished curve of the geometry if $X \in \mathfrak{g}_{-}$.

- A distinguished chart on $M$ is a chart with values in $\mathfrak{g}_{-}$obtained as a local inverse of $\left.\pi \circ \exp _{b}\right|_{\mathfrak{g}_{-}}$.

For a given parabolic model, exponential and distinguished curves can be classified by the geometric type of the initial direction (see section 1.2 below). Section 5.3 of [4] contains thorough descriptions of the classes of distinguished curves for many parabolic models. In the next section, we introduce normal charts, with values in tangent spaces.

1.1.2. Adjoint tractor bundle and infinitesimal automorphisms. Natural vector bundles on parabolic geometries modeled on $G / P$ can be obtained as associated bundles to the Cartan bundle. Given a representation $\mathbb{W}$ of $P$, form $B \times{ }_{P} \mathbb{W}$. Using the Cartan connection, such bundles can sometimes be identified with tensor bundles; for example, the adjoint representation restricted to $P$ descends to the quotient vector space $\mathfrak{g} / \mathfrak{p}$, and $B \times_{P}(\mathfrak{g} / \mathfrak{p}) \cong T M$. The main idea to define this isomorphism is to note that for each $b \in B$ with $\pi(b)=x \in M$, the linear isomorphism $\omega_{b}: T_{b} B \rightarrow \mathfrak{g}$ induces a linear isomorphism $T_{x} M \cong T_{b} B / \operatorname{ker}\left(D_{b} \pi\right) \rightarrow \mathfrak{g} / \mathfrak{p}$.

For $b \in B$ with $\pi(b)=x \in M$, the linear isomorphism $T_{x} M \rightarrow \mathfrak{g} / \mathfrak{p} \rightarrow$ $\mathfrak{g}_{-}$composed with the normal coordinate chart $\pi \circ \exp _{b}$ gives a diffeomorphism from an open neighborhood of 0 in $T_{x} M$ onto an open 
neighborhood of $x \in M$, which we will refer to as a normal coordinate chart for $M$. By construction, a straight line $\{t \xi: t \in \mathbf{R}\}$ through $0 \in T_{x} M$ corresponds to a distinguished curve through $x$ with initial direction $\xi$.

Two further associated bundles to the Cartan bundle will play a role in the sequel. The Killing form of $\mathfrak{g}$ gives an identification of $(\mathfrak{g} / \mathfrak{p})^{*}$ with $\mathfrak{p}_{+}$, so $B \times_{P} \mathfrak{p}_{+} \cong T^{*} M$. The adjoint tractor bundle is $\mathcal{A} M=B \times_{P} \mathfrak{g}$ and is useful for studying infinitesimal automorphisms. The filtration of $\mathfrak{g}$ gives rise to a filtration $\mathcal{A} M=\mathcal{A}^{-k} M \supset \cdots \supset \mathcal{A}^{k} M$ by smooth subbundles. Since $\mathfrak{g}^{1}=\mathfrak{p}_{+}$and $\mathfrak{g}^{0}=\mathfrak{p}$, we see that $\mathcal{A}^{1} M \cong T^{*} M$ and $\mathcal{A} M / \mathcal{A}^{0} M \cong T M$. Denote by $\Pi: \mathcal{A} M \rightarrow T M$ the resulting natural projection.

The curvature of $\omega$ from definition 1.2 can be naturally viewed as an element $\kappa \in \Omega^{2}(M, \mathcal{A} M)$. Indeed, from the defining properties of $\omega$ it follows easily that $K \in \Omega^{2}(B, \mathfrak{g})$ is horizontal and $P$-equivariant and thus corresponds to a form $\kappa$ as above.

Vector fields on $B$ are in bijective correspondence with $\mathfrak{g}$-valued smooth functions via $\xi \mapsto \omega(\xi)$. Equivariance of $\omega$ immediately implies that $\omega(\xi)$ is a $P$-equivariant function if and only if $\xi$ is a right- $P$-invariant vector field. The space $\Gamma(\mathcal{A} M)$ of smooth sections of $\mathcal{A} M$ can be naturally identified with the space $\mathfrak{X}(B)^{P}$ of $P$-invariant vector fields on $B$; note that these descend to $M$. On the bundle $\mathcal{A} M$, the corresponding projection is $\Pi$.

An automorphism of $(M, B, \omega)$ is a principal bundle automorphism that preserves $\omega$. These form a Lie group, which will be denoted $\operatorname{Aut}(M, B, \omega)$. An infinitesimal automorphism is given by $\xi \in \mathfrak{X}(B)^{P}$ such that $\mathcal{L}_{\xi} \omega=0$, where $\mathcal{L}$ denotes the Lie derivative. (For a pseudoRiemannian metric, infinitesimal automorphisms are called Killing fields.) An infinitesimal automorphism $\tilde{\eta}$ descends to a vector field $\eta$ on $M$. The resulting subalgebra of $\mathfrak{X}(M)$ will be denoted $\mathfrak{i n f}(M)$ below; note these vector fields are not assumed to be complete.

1.1.3. Normal parabolic geometries and harmonic curvature. Parabolic geometries encode certain underlying geometric structures. First note 
that via the isomorphism $T M \cong \mathcal{A} M / \mathcal{A}^{0} M$, a parabolic geometry of type $(\mathfrak{g}, P)$ gives rise to a filtration $T M=T^{-k} M \supset \cdots \supset T^{-1} M$ of the tangent bundle, where $T^{i} M=\mathcal{A}^{i} M / \mathcal{A}^{0} M$. This filtration gives rise to a filtration of $\Omega^{2}(M, \mathcal{A} M)$ by homogeneity: $\tau \in \Omega^{2}(M, \mathcal{A} M)$ is called homogeneous of degree $\geq \ell$ if for $\xi \in T^{i} M$ and $\eta \in T^{j} M$ the value $\tau(\xi, \eta) \in \mathcal{A}^{i+j+\ell} M$

The geometry $(M, B, \omega)$ is regular if the curvature 2-form $\kappa$ is homogeneous of degree at least 1. A Cartan geometry is torsion-free if $\kappa$ has values in $\mathcal{A}^{0} M \subset \mathcal{A} M$; torsion-free implies regular. Now assuming regularity, the underlying structure of a parabolic geometry consists of the filtration $\left\{T^{i} M\right\}_{i \in \mathbf{N}}$ of the tangent bundle and a reduction of structure group of the associated graded of this filtered bundle to $G_{0}$. Conversely, any such structure of a filtration with a $G_{0}$-reduction can be obtained from some regular parabolic geometry.

These geometric structures are equivalent, in the categorical sense, to regular parabolic geometries satisfying an additional condition on $\kappa$ called normality. The Lie algebra homology differentials for the Lie algebra $\mathfrak{p}_{+}$with coefficients in the module $\mathfrak{g}$ defines a $P$-equivariant homomorphism $\Lambda^{k} \mathfrak{p}_{+} \otimes \mathfrak{g} \rightarrow \Lambda^{k-1} \mathfrak{p}_{+} \otimes \mathfrak{g}$, which is traditionally denoted by $\partial^{*}$ and called the Kostant codifferential. For $k=2$, this homomorphism gives on the level of associated bundles a natural bundle map $\Lambda^{2} T^{*} M \otimes \mathcal{A} M \rightarrow T^{*} M \otimes \mathcal{A} M$, also denoted by $\partial^{*}$. Now the geometry is called normal if $\partial^{*} \kappa=0$.

The projective and conformal structures mentioned above correspond to $|1|$-gradings. Other geometric structures arising from parabolic Cartan geometries include almost-Grassmannian and almost-quaternionic structures, hypersurface-type CR structures, path geometries, and several types of generic distributions.

The equivalence in the categorical sense implies that any automorphism of the underlying structure uniquely lifts to an automorphism of the parabolic geometry. The analogous result for vector fields says any infinitesimal automorphism $\eta$ of the underlying structure lifts uniquely to $\tilde{\eta} \in \mathfrak{X}(B)^{P}$ such that $\mathcal{L}_{\tilde{\eta}} \omega=0$. Conversely, projecting an infinitesimal 
automorphism of $(M, B, \omega)$ to $M$ gives an infinitesimal automorphism of the underlying structure.

The normality condition for parabolic geometries can also be used to extract the essential part of the curvature of the canonical Cartan connection. As mentioned above, the Kostant codifferential induces bundle maps

$$
\Lambda^{3} T^{*} M \otimes \mathcal{A} M \rightarrow \Lambda^{2} T^{*} M \otimes \mathcal{A} M \rightarrow T^{*} M \otimes \mathcal{A} M
$$

Since these maps come from a homology differential, the composition of the two bundle maps above is zero, so there are natural subbundles $\operatorname{im}\left(\partial^{*}\right) \subset \operatorname{ker}\left(\partial^{*}\right) \subset \Lambda^{2} T^{*} M \otimes \mathcal{A} M$. By construction, the quotient bunde $\mathcal{H}_{2}=\operatorname{ker}\left(\partial^{*}\right) / \operatorname{im}\left(\partial^{*}\right)$ can be realized as $B \times_{P} H_{2}\left(\mathfrak{p}_{+}, \mathfrak{g}\right)$, and the latter Lie algebra homology group can can identified with the Lie algebra cohomology group $H^{2}\left(\mathfrak{g}_{-}, \mathfrak{g}\right)$. For a regular normal parabolic geometry, the curvature $\kappa$ actually is a section of $\operatorname{ker}\left(\partial^{*}\right)$. The harmonic curvature $\kappa_{H} \in \Gamma\left(\mathcal{H}_{2}\right)$ is the image of $\kappa$ under the obvious quotient projection.

General theorems assert that no information is lost in passing from $\kappa$ to $\kappa_{H}$. First, vanishing of $\kappa_{H}$ on an open subset $U \subset M$ implies vanishing of $\kappa$ on $U$. In fact, there is a natural differential operator $S: \Gamma\left(\mathcal{H}_{2}\right) \rightarrow \Omega^{2}(M, \mathcal{A} M)$ such that $S\left(\kappa_{H}\right)=\kappa$. The crucial advantage of the harmonic curvature is that one can show that the representation $H_{2}\left(\mathfrak{p}_{+}, \mathfrak{g}\right)$ is always completely reducible, so the corresponding associated bundle is a simpler geometric object than $\Lambda^{2} T^{*} M \otimes \mathcal{A} M$. The structure of $H_{2}\left(\mathfrak{p}_{+}, \mathfrak{g}\right)$ can be computed with Kostant's version of the Bott-Borel-Weil theorem-see section 3.3 of [4]. We will use without further citation the resulting descriptions of harmonic curvature components for the individual geometries we discuss.

\subsection{Higher order fixed points and the main questions. Let} $(M, B, \omega)$ be a regular normal parabolic geometry of type $(\mathfrak{g}, P)$. Let $\eta \in \mathfrak{i n f}(M)$, and denote the induced vector field on $B$ by $\tilde{\eta}$ and by $s$ the corresponding section of $\mathcal{A} M$. For $x_{0} \in M$, we have $\eta\left(x_{0}\right)=0$ if and only if $s\left(x_{0}\right) \in \mathcal{A}^{0} M$, equivalently if $\omega_{b_{0}}(\tilde{\eta}) \in \mathfrak{p}$ for any $b_{0} \in \pi^{-1}\left(x_{0}\right)$. 
Definition 1.5. The infinitesimal automorphism $\eta$, or the corresponding section $s$ of $\mathcal{A} M$, has a higher order fixed point at $x_{0}$ if $s\left(x_{0}\right) \in$ $\mathcal{A}^{1} M$. In this case, the isotropy at $x_{0}$ of $\eta$ (or of $s$ ) is the element of the cotangent space corresponding to $s\left(x_{0}\right)$.

Via $b_{0} \in \pi^{-1}\left(x_{0}\right)$ the isotropy $\alpha \in T_{x_{0}}^{*} M$ corresponds to an element in $\mathfrak{p}_{+}$. The geometric type of the infinitesimal automorphism at $x_{0}$ is defined to be the $P$-orbit of this element, which is independent of the choice of $b_{0}$. These geometric types give rise to an initial classification of higher-order fixed points. For example, in the conformal case, there is a natural inner product on $T_{x_{0}}^{*} M$ up to scale, so isotropies can be postive, null, or negative. For algebraically more complicated models, as for CR structures, the cotangent bundle has a natural filtration induced by $\mathcal{A}^{1} M \supset \cdots \supset \mathcal{A}^{k} M$, which leads to a variety of possible geometric types of isotropies. These will be discussed in detail in several examples below. In all cases we consider there are only finitely many orbits, and in general there are finiteness results of E. Vinberg [7].

Main Questions: What special dynamical properties are shared by infinitesimal automorphisms admitting a higher order fixed point with isotropy of a certain geometric type? What curvature restrictions are implied by existence of such an automorphism? Which types of higher order fixed points in $x_{0}$ imply that $(M, B, \omega)$ is locally flat on a nonempty open set $U$ with $x_{0} \in \bar{U}$ ?

Remark 1.6. The concept of essential automorphisms, which previously existed for conformal and projective structures, has recently been extended to all parabolic geometries by J. Alt in [8]. It is immediate from his proposition 3.4 that a Killing field with a higher order fixed point is essential.

1.3. Results. We develop general tools in section 2 that give the precise action of a flow on specific curves emanating from a higher order fixed point. Several of these propositions generalize the tools of [1] and [9]. We can apply them to recover the previously cited theorems on higher order fixed points for projective and conformal flows. These applications are presented in a separate article [10]. 
In section 3.1, we apply these tools to $(2, n)$-almost-Grassmannian structures to describe the two types of higher order fixed points in these geometries, and we show in theorem 3.1 that if the geometry is torsionfree, then existence of either type implies flatness on a nonempty open set. The proofs in this section easily adapt to prove an analogous result for almost-quaternionic structures, without any torsion-freeness assumption (see theorem 3.7).

Next we prove a general result for parabolic contact structures (see section 3.3): another natural generalization of the hypotheses in the projective and conformal results [1] and [3] is to assume that a flow fixes a point $x_{0}$ and has trivial derivative at $x_{0}$, which in the parabolic contact case is a stronger assumption than $x_{0}$ being a higher order fixed point. Under this hypothesis, we prove in theorem 3.9 that the curvature vanishes on an open set with $x_{0}$ in its closure; in some cases, we can further deduce flatness on a neighborhood of $x_{0}$.

Finally, in section 3.4, we treat partially integrable almost-CR structures. Theorem 3.11 says the harmonic curvature always vanishes at a higher order fixed point, and certain types of higher order fixed points imply flatness on a nonempty open set. A consequence of this theorem is a local $C^{\omega}$ version of the Schoen-Webster theorem on automorphisms of strictly pseudoconvex CR structures (see theorem 3.16; compare also [11] and [12].). For general nondegenerate CR structures, we are left with the following question. (Beloshapka [13] and Loboda [14 have proved the answer is no in the case of real-analytic hypersurfaces of complex manifolds.)

Question 1. Is there a non-flat partially integrable almost-CR manifold, the automorphism group of which has a higher-order fixed point?

\section{General Results}

We note that the holonomy calculations of propositions 2.5 and 2.12 below generalize lemma 5.5 of [1] from the projective setting and proposition 4.5 of 9 ] from the conformal case. 
We begin with a basic proposition from [9] that computes the holonomy of an automorphism of a Cartan geometry with isotropy $g$ in terms of the action of $g$ on $G$.

Proposition 2.1. [9, prop 4.3] Let $\varphi \in \operatorname{Aut}(M, B, \omega)$. Suppose

- $\varphi b_{0}=b_{0} g$ for some $b_{0} \in B$ and $g=g_{0} \in P$

- $\exp \left(b_{0}, s U\right)$ is defined for $s$ in an interval $I$ around 0 and for some $U \in \mathfrak{g}$

- $g e^{s U}=e^{c(s) U} p(s)$ in $G$, where $p(t): I \rightarrow P$ with $p(0)=g_{0}$, and $c: I \rightarrow I^{\prime}$ is a diffeomorphism fixing 0 .

Then the corresponding equation holds in $B: \exp \left(b_{0}, s U\right)$ is also defined on $I^{\prime}$, and

$$
\varphi \exp \left(b_{0}, s U\right)=\exp \left(b_{0}, c(s) U\right) p(s)
$$

Remark 2.2. The proposition says that in normal coordinates for $(M, B, \omega)$ centered at the fixed point $\pi\left(b_{0}\right)$, the automorphism $\varphi$ resembles the model automorphism $g$ acting on $G / P$ with fixed point $o=[P]$. The proposition is related to the comparison maps studied in the recent paper [15].

Before stating our first general results on higher order fixed points, we introduce some terminology.

Definition 2.3. Let $\eta \in \mathfrak{i n f}(M)$.

- The strongly fixed set of a given geometric type of the flow generated by $\eta$ is the set of all higher order fixed points of that type. For a higher order fixed point $x_{0}$, the term strongly fixed set will mean the strongly fixed set of the type of $x_{0}$.

- Given a neighborhood $U$ of a higher order fixed point $x_{0}$ the strongly fixed component of $x_{0}$ in $U$ is the set of all points that can be reached from $x_{0}$ by a smooth curve contained in the intersection of $U$ with the strongly fixed set of $x_{0}$. The higher order fixed point $x_{0}$ is called smoothly isolated if $\left\{x_{0}\right\}$ equals the strongly fixed component in some neighborhood. 
For any $x_{0} \in M$ and any choice of $b \in \pi^{-1}\left(x_{0}\right)$, the Cartan connection $\omega$ gives identifications $T_{x_{0}}^{*} M \cong \mathfrak{p}_{+}$and $T_{x_{0}} M \cong \mathfrak{g} / \mathfrak{p}$ such that the duality between the two spaces is induced by the Killing form of $\mathfrak{g}$. Then any $\alpha \in T_{x_{0}}^{*} M$ corresponds to an element $Z \in \mathfrak{p}_{+}$. Put

$$
\begin{gathered}
F_{\mathfrak{g}}(Z)=\left\{X \in \mathfrak{g}: \operatorname{ad}_{X}^{k}(Z) \in \mathfrak{p} \quad \forall k \in \mathbf{N}\right\} \\
C_{\mathfrak{g}}(Z)=\{X \in \mathfrak{g}:[X, Z]=0\} \subset F_{\mathfrak{g}}(Z)
\end{gathered}
$$

A different choice $b^{\prime} \in \pi^{-1}\left(x_{0}\right)$ is of the form $b^{\prime}=b \cdot g$ for some $g \in P$, which leads to $Z^{\prime}=\operatorname{Ad}\left(g^{-1}\right)(Z) ; F_{\mathfrak{g}}\left(Z^{\prime}\right)=\operatorname{Ad}\left(g^{-1}\right)\left(F_{\mathfrak{g}}(Z)\right) ; C_{\mathfrak{g}}\left(Z^{\prime}\right)=$ $\operatorname{Ad}\left(g^{-1}\right)\left(C_{\mathfrak{g}}(Z)\right)$. By point 2(b) in definition 1.1 the images of these subsets in $\mathfrak{g} / \mathfrak{p}$ determine subsets $C(\alpha) \subset F(\alpha) \subset T_{x_{0}} M$ which are independent of the choice of $b$. Of course, $C(\alpha) \subset T_{x_{0}} M$ is a linear subspace.

Definition 2.4. The subset $F(\alpha) \subset T_{x_{0}} M$ and the subspace $C(\alpha) \subset$ $T_{x_{0}} M$ determined by $\alpha \in T_{x_{0}}^{*} M$ as above are called, respectively, the normalizing subset and the commutant of $\alpha$ in $T_{x_{0}} M$.

The following is a consequence of proposition 2.1 for the local behavior of an infinitesimal automorphism around a higher order fixed point.

Proposition 2.5. Consider a Cartan geometry $(M, B, \omega)$ modeled on $(\mathfrak{g}, P)$ and $\eta \in \mathfrak{i n f}(M)$ with higher order fixed point $x_{0} \in M$ with isotropy $\alpha \in T_{x_{0}}^{*}(M)$.

(1) For any $\xi \in F(\alpha) \subset T_{x_{0}} M$, there is an exponential curve emanating from $x_{0}$ in the direction $\xi$ consisting of fixed points for $\eta$. If $\xi \in C(\alpha) \subset F(\alpha)$, then this curve lies in the strongly fixed set of $x_{0}$.

(2) If $k=\operatorname{dim} C(\alpha)$, then there is a $k$-dimensional submanifold $N \subset M$ through $x_{0}$ contained in the strongly fixed set of $x_{0}$, with $T_{x_{0}} N=C(\alpha)$.

(3) For some neighborhood $V$ of $x_{0}$ any point in the strongly fixed component of $x_{0}$ in $V$ can be reached by an exponential curve emanating from $x_{0}$ in a direction belonging to $C(\alpha)$. In particular, if $C(\alpha)=\{0\}$, then $x_{0}$ is smoothly isolated. 
Proof: Choose $b_{0} \in \pi^{-1}\left(x_{0}\right)$ and set $Z=\omega_{b_{0}}(\tilde{\eta}) \in \mathfrak{p}_{+}$. Let $U$ be a neighborhood of 0 in $\mathfrak{g}$ on which the restriction of $\exp _{b_{0}}$ is defined and is a diffeomorphism onto its image. For $\xi \in F(\alpha)$, there is an element $X \in F_{\mathfrak{g}}(Z)$ such that $D_{b_{0}} \pi\left(\omega^{-1}(X)\right)=\xi$. Let $\gamma(r)=\pi\left(\exp _{b_{0}}(r X)\right)$, defined for small $r$.

In $G$ we have $e^{t Z} e^{r X}=e^{r X} e^{t \operatorname{Ad}\left(e^{-r X}\right)(Z)}$ and

$$
\operatorname{Ad}\left(e^{-r X}\right)(Z)=\sum_{k=0}^{\infty} \frac{(-r)^{k}}{k !} \operatorname{ad}_{X}^{k}(Z),
$$

which lies in $\mathfrak{p}$ since $X \in F_{\mathfrak{g}}(Z)$. If $\xi \in C(\alpha)$, then $X$ can be chosen in $C_{\mathfrak{g}}(Z)$, and then $\operatorname{Ad}\left(e^{-r X}\right)(Z)=Z$. It follows from proposition 2.1 that in $B$, for all sufficiently small $t, X \in V$, and $r \leq 1$,

$$
\varphi_{\tilde{\eta}}^{t} \exp \left(b_{0}, r X\right)=\exp \left(b_{0}, r X\right) e^{t \operatorname{Ad}\left(e^{-r X}\right)(Z)}
$$

Now (1) follows for $F(\alpha)$ because the rightmost term is in $P$. For $\xi \in$ $C(\alpha)$, differentiate with respect to $t$ at time 0 to see that $\tilde{\eta}\left(\exp \left(b_{0}, r X\right)\right)$ coincides with the fundamental vector field generated by $Z$ for all $r$.

For point (2), note that we can choose a $k$-dimensional subspace $C \subset$ $C_{\mathfrak{g}}(Z)$ with $C \cap \mathfrak{p}=0$. Extend $C$ to a linear subspace of $\mathfrak{g}$ which is complementary to $\mathfrak{p}$. Then $\pi \circ \exp _{b_{0}}$ can be restricted to an open neighborhood of zero to obtain a submanifold chart as required.

For (3), let $\gamma(r)$ be a smooth curve emanating from $x_{0}$ which lies in the strongly fixed set of $x_{0}$. Then there is a lift $\tilde{\beta}$ of $\gamma$, which we may assume begins at $b_{0}$, satisfying

$$
\tilde{\eta}_{\tilde{\beta}(r)}=\left(\tilde{Y}_{r}\right)_{\tilde{\beta}(r)},
$$

for some $Y_{r} \in \mathfrak{p}$ with corresponding fundamental vector field $\tilde{Y}_{r}$; moreover, each $Y_{r}$ is conjugate in $P$ to $Z$, and $Y_{0}=Z$. Now the $P$-conjugacy class of $Z$ is in bijection with $P / C_{P}(Z)$, where $C_{P}(Z)$ is the stabilizer in $P$ of $Z$ under the adjoint action. A smooth path in this quotient can be lifted to $P$. Hence on a sufficiently small interval, we obtain a smooth path $c_{r}$ through the identity in $P$ such that $\operatorname{Ad}\left(c_{r}\right)\left(Y_{r}\right)=Z$. Then let $\tilde{\gamma}=\tilde{\beta} c_{r}^{-1}$. For this lift, 


$$
\tilde{\eta}_{\tilde{\gamma}(r)}=\left(\widehat{\operatorname{Ad}\left(c_{r}\right)}\left(Y_{r}\right)\right)_{\tilde{\beta}(r)}=\tilde{Z}_{\tilde{\beta}(r)}
$$

Therefore $\varphi_{\tilde{\eta}}^{t} \tilde{\gamma}(r)=\tilde{\gamma}(r) e^{t Z}$ for sufficiently small $r$. Fix $r$ for which $\tilde{\gamma}(r) \in \exp _{b_{0}}(U)$, so there is $X \in U$ such that $\exp _{b_{0}}(X)=\tilde{\gamma}(r)$. Then

$$
\varphi_{\tilde{\eta}}^{t} \exp _{b_{0}}(X)=\exp _{b_{0}}(X) e^{t Z}
$$

for all $t$. On the other hand, the expression above also equals

$$
\exp _{b_{0} e^{t Z}}(X)=\exp _{b_{0}}\left(\operatorname{Ad}\left(e^{t Z}\right)(X)\right) e^{t Z}
$$

Therefore $\exp _{b_{0}}(X)=\exp _{b_{0}}\left(\operatorname{Ad}\left(e^{t Z}\right)(X)\right)$, so for any $t$ sufficiently small that $\operatorname{Ad}\left(e^{t Z}\right)(X) \in U$, we have $\operatorname{Ad}\left(e^{t Z}\right)(X)=X$, and thus $[Z, X]=0$, which completes the proof of $(3)$. $\diamond$

Remark 2.6. In most cases we discuss, we can strengthen this result by showing that the curves in (1) are distinguished curves for the geometry and describing the submanifold in (2) in terms of normal coordinates. These improvements will be presented at the end of section 2 .

To proceed further, we need an analog of the concept of holonomy sequences associated to sequences of automorphisms of a Cartan geometry, which appeared in [16]. The notion was further developed in later papers, including [17], [9], [2], and [18]. The following definition for flows is the most useful variation for our purposes.

Definition 2.7. Let $\left\{\varphi^{t}\right\}$ be a flow by automorphisms of $(M, B, \omega)$, and let $b \in B$. A path $p(t) \in P$ is a holonomy path at $b$ with attractor $b_{0} \in B$ for $\left\{\varphi^{t}\right\}$ if there exists a path $b(t)$ and a point $b_{0}$ in $B$ with

$$
b(t) \rightarrow b \quad \text { and } \quad \varphi^{t} b(t) p(t)^{-1} \rightarrow b_{0}
$$

as $t \rightarrow \infty$.

The following proposition provides a condition under which a holonomy path at one point can be propagated to nearby points.

Proposition 2.8. Let $\left\{\varphi^{t}\right\}$ be a flow by automorphisms of $(M, B, \omega)$. Let $p(t)$ be a holonomy path at $b$ with attractor $b_{0}$ and path $b(t)$ as in definition 2.7. Suppose that for some $Y \in \mathfrak{g}$, $\exp (b(t), Y)$ is defined for 
all $t$ and $A d(p(t))(Y) \rightarrow Y_{\infty}$ as $t \rightarrow \infty$. Then $p(t)$ is a holonomy path at $\exp (b, Y)$ with attractor $\exp \left(b_{0}, Y_{\infty}\right)$.

Proof: We have

$$
\varphi^{t} b(t) p(t)^{-1}=k(t) \rightarrow b_{0}
$$

Then $\exp (b(t), Y) \rightarrow \exp (b, Y)$, and

$$
\begin{aligned}
\varphi^{t} \exp (b(t), Y) p(t)^{-1} & =\exp \left(\varphi^{t} b(t), Y\right) p(t)^{-1} \\
& =\exp (k(t) p(t), Y) p(t)^{-1} \\
& =\exp (k(t), \operatorname{Ad}(p(t)) Y) \rightarrow \exp \left(b_{0}, Y_{\infty}\right)
\end{aligned}
$$

$\diamond$

A holonomy path $p(t)$ at $b$ leads to restrictions on the possible $\varphi^{t}$ invariant sections of any bundle associated to the Cartan bundle. Given a representation $\mathbb{W}$ of $P$, a principal bundle automorphism $\varphi$ of $B$ gives rise to an automorphism of the associated bundle $B \times_{P} \mathbb{W}$. If $\varphi$ also preserves $\omega$, and the associated bundle is a tensor bundle, then this automorphism is the one functorially associated to $\varphi$. Smooth sections of $B \times{ }_{P} \mathbb{W}$ correspond to smooth $P$-equivariant maps $f: B \rightarrow \mathbb{W}$ that is, $f(b g)=g^{-1} f(b)$ for any $g \in P$. The pullback of a section by $\varphi$ corresponds to the precomposition of $f: B \rightarrow \mathbb{W}$ with $\varphi$. In particular, if $f$ corresponds to a $\varphi^{t}$-invariant section for a flow, then $f \circ \varphi^{t}=f$ for all $t$.

The Cartan curvature $\kappa$, and components of the harmonic curvature for parabolic geometries, are both invariant under automorphisms because the corresponding sections are constructed naturally from the Cartan connection. For the Cartan curvature, the representation $\mathbb{W}$ is $\Lambda^{2}(\mathfrak{g} / \mathfrak{p})^{*} \otimes \mathfrak{g}$, which in general is rather complicated; for the components of the harmonic curvature, in contrast, the representation is always irreducible. The number of harmonic curvature components and the form of the corresponding representations varies according to the type of geometry in question.

Proposition 2.9. Let $p(t)$ be a holonomy path for $\left\{\varphi^{t}\right\}$ corresponding to $b(t) \rightarrow b$ with attractor $b_{0}$, and let $f: B \rightarrow \mathbb{W}$ be the equivariant 
function corresponding to a $\varphi^{t}$-invariant section of $B \times_{P} \mathbb{W}$. Put $k(t)=$ $\varphi^{t}(b(t)) p(t)^{-1}$ as in definition 2.7 .

(1) Then $p(t) \cdot f(b(t)) \rightarrow f\left(b_{0}\right)$ as $t \rightarrow \infty$.

(2) Assume moreover that $p(t)$ is contained in a 1-parameter subgroup of $P$ that is diagonalizable on $\mathbb{W}$, and let $\mathbb{W}=\mathbb{W}_{0} \oplus$ $\cdots \oplus \mathbb{W}_{\ell}$ be an eigenspace decomposition with eigenvalues given by functions $\lambda_{i}(t), i=1, \ldots, \ell$. Let $f(b)_{i}$ be the component of $f(b)$ in $\mathbb{W}_{i}$.

- If $\lambda_{i}(t) \rightarrow \pm \infty$ as $t \rightarrow \infty$, then $f(b)_{i}=0$.

- If $f(b)_{i} \neq 0$, then $\left\|f(k(t))_{i}\right\| \in \Theta\left(\lambda_{i}(t)\right)$, where $\|\cdot\|$ is any norm on $\mathbb{W}$; in particular, if $f\left(b_{0}\right)_{i}=0$ but $\lambda_{i}(t)$ does not tend to 0 as $t \rightarrow \infty$, then $f(b)_{i}=0$.

Recall that for functions $f$ and $g$ defined on $\mathbf{R}^{+}$, the notation $f(t) \in$ $\Theta(g(t))$ means there exist nonzero constants $c, C \in \mathbf{R}^{+}$such that

$$
c g(t) \leq f(t) \leq C g(t) \quad \text { for all } \quad t \geq 0
$$

\section{Proof:}

For (1), we see that the $P$-equivariance of $f$ implies

$$
f(k(t))=p(t) \cdot f\left(\varphi^{t}(b(t))\right),
$$

and $\varphi^{t}$-invariance implies $f\left(\varphi^{t}(b(t))\right)=f(b(t))$; now (1) follows.

For (2) we have by (1) that

$$
\lambda_{i}(t) f(b(t))_{i} \rightarrow f\left(b_{0}\right)_{i} \quad \text { for } t \rightarrow \infty
$$

Since $f(b(t)) \rightarrow f(b)$ for $t \rightarrow \infty$, the first property follows immediately. For the second, also use that $f(k(t))_{i}=\lambda_{i}(t) f(b(t))_{i} \cdot \diamond$

Definition 2.10. Let $p(t)$ be a holonomy path at $b$ and $\mathbb{W}$ a P-representation satisfying the hypotheses of part (2) of proposition $\left[2.9\right.$ above. Let $\mathbb{W}_{i}$ and $\lambda_{i}(t), i=0, \ldots, l$, be as above.

- The stable subspace for $p$, denoted $\mathbb{W}_{s t}(p)$, is the sum of the eigenspaces $\mathbb{W}_{i}$ for which $\lambda_{i}(t)$ is bounded as $t \rightarrow \infty$. 
- The strongly stable subspace for $p$, denoted $\mathbb{W}_{s s}(p)$, is the sum of eigenspaces $\mathbb{W}_{i}$ with $\lambda_{i}(t) \rightarrow 0$ as $t \rightarrow \infty$.

Part (2) of proposition 2.9 says that we must always have $f(b) \in$ $\mathbb{W}_{s t}(p)$, and if $f\left(b_{0}\right)=0$, then also $f(b) \in \mathbb{W}_{s s}(p)$.

A crucial fact for the sequel is that in the case of parabolic geometries, nice holonomy paths can be obtained from purely algebraic data. Recall that an $\mathfrak{s l}_{2}$-triple in a Lie algebra $\mathfrak{a}$ is formed by elements $E, H, F \in \mathfrak{a}$ such that $[E, F]=H,[H, E]=2 E$, and $[H, F]=-2 F$. The JacobsonMorozov theorem says that any element $E$ in the semisimple Lie algebra $\mathfrak{g}$ which is nilpotent in the adjoint representation can be completed to an $\mathfrak{s l}_{2}$-triple: there exists $F \in \mathfrak{g}$ such that $E,[E, F]$, and $F$ form an $\mathfrak{s l}_{2}$-triple.

Let $x_{0} \in M$ and $\alpha \in T_{x_{0}}^{*} M$. As above, a choice of $b_{0} \in \pi^{-1}\left(x_{0}\right)$ associates to $\alpha$ an element $Z \in \mathfrak{p}_{+}$, which is nilpotent. We define $T_{\mathfrak{g}}(Z)$ to be the non-empty set of all $X \in \mathfrak{g}$, such that $Z,[Z, X]$, and $X$ form an $\mathfrak{s l}_{2}$-triple. As for the normalizing set and the commutant, we obtain a subset $T(\alpha) \subset T_{x_{0}} M$ which is independent of the choice of $b$.

Definition 2.11. For $\alpha \in T_{x_{0}}^{*} M$, the non-empty subset $T(\alpha) \subset T_{x_{0}} M$ defined above is called the counterpart set of $\alpha$.

Now we can precisely compute the action of $\varphi^{t}$ on certain curves from $x_{0}$ in any direction belonging to the counterpart set of the isotropy.

Proposition 2.12. Let $(M, B, \omega)$ be modeled on $(\mathfrak{g}, P)$, and let $\eta \in$ $\mathfrak{i n f}(M)$ have a higher order fixed point at $x_{0} \in M$ with isotropy $\alpha \in$ $T_{x_{0}}^{*} M$. Let $\xi \in T(\alpha)$, and let $Z, X \in \mathfrak{g}$ be the elements associated to $\alpha$ and $\xi$, respectively, for a choice of $b_{0} \in \pi^{-1}\left(x_{0}\right)$; set $A=[Z, X]$.

Then there exists an exponential curve $\sigma:(-\epsilon, \epsilon) \rightarrow M$, for some $\epsilon>0$, such that $\sigma(0)=x_{0}, \sigma^{\prime}(0)=\xi$, and

$$
\varphi_{\eta}^{t}(\sigma(s))=\sigma\left(\frac{s}{1+s t}\right) \text { whenever }|s|<\epsilon \text { and } t s \geq 0
$$

Moreover, for each such $s$, there is $b \in \pi^{-1}(\sigma(s))$ such that $g(t)=$ $e^{\log (1+s t) A}$ is a holonomy path at $b$ with attractor $b_{0}$. 
Proof: In $S L(2, \mathbf{R})$, compute

$$
\left(\begin{array}{ll}
1 & t \\
0 & 1
\end{array}\right)\left(\begin{array}{ll}
1 & 0 \\
s & 1
\end{array}\right)=\left(\begin{array}{cc}
1 & 0 \\
\frac{s}{1+s t} & 1
\end{array}\right)\left(\begin{array}{cc}
1+s t & 0 \\
0 & \frac{1}{1+s t}
\end{array}\right)\left(\begin{array}{cc}
1 & \frac{t}{1+s t} \\
0 & 1
\end{array}\right) .
$$

The $\mathfrak{s l}_{2}$-triple in $\mathfrak{g}$ formed by $Z, A$, and $X$ gives rise to a Lie algebra homomorphism $\mathfrak{s l}(2, \mathbf{R}) \rightarrow \mathfrak{g}$, which locally integrates to a group homomorphism $S L(2, \mathbf{R}) \rightarrow G$. The above equation then shows that in G

$$
e^{t Z} e^{s X}=e^{\frac{s}{1+s t} X} e^{\log (1+s t) A} e^{\frac{t}{1+s t} Z} .
$$

Proposition 2.1 shows that the local flow $\varphi_{\tilde{\eta}}^{t}$ of $\tilde{\eta}$ satisfies

$$
\varphi_{\tilde{\eta}}^{t} \exp \left(b_{0}, s X\right)=\exp \left(b_{0}, \frac{s}{1+s t} X\right) e^{\log (1+s t) A} e^{\frac{t}{1+s t} Z}
$$

for sufficiently small $s$. Fixing such a value for $s$, the path $b(t)=$ $\exp \left(b_{0}, s X\right) e^{\frac{-t}{1+s t} Z}$ evidently satsfies $b(t) \rightarrow b=\exp \left(b_{0}, s X\right) e^{-\frac{1}{s} Z}$, and $\pi(b)=\pi\left(\exp \left(b_{0}, s X\right)\right)$. Define $\sigma(s)$ to be this latter curve, valid on some nonzero interval $(-\epsilon, \epsilon)$.

Note $\sigma^{\prime}(0)=D_{b_{0}} \pi\left(\omega_{b_{0}}^{-1}(X)\right)=\xi$, and $\sigma^{\prime}(s) \neq 0$ for all $|s|<\epsilon$. Finally, equation (11) says that $\varphi_{\tilde{\eta}}^{t}(b(t)) \cdot e^{-\log (1+s t) A}=\exp \left(b_{0}, \frac{s}{1+s t} X\right)$, which converges to $b_{0}$ for $t s>0$ as $|t| \rightarrow \infty$. $\diamond$

Basic representation theory says that in a standard basis of $\mathfrak{s l}(2, \mathbf{R})$, the semisimple element $H$ acts diagonalizably with integer eigenvalues in any finite-dimensional complex representation. Thus for an $\mathfrak{s l}_{2}$-triple $Z, A, X$ in $\mathfrak{g}$, the endomorphism $\operatorname{ad}(A)$ is diagonalizable on $\mathfrak{g}$, so $A \in \mathfrak{g}$ is a semisimple element. Further, $A$ acts diagonalizably with integer eigenvalues on any finite-dimensional complex representation of $\mathfrak{g}$. We will assume all representations are finite-dimensional below.

If we assume in addition that $A \in \mathfrak{g}_{0}$, then $\operatorname{ad}(A)\left(\mathfrak{g}_{0}\right) \subset \mathfrak{g}_{0}$, $\operatorname{so} \operatorname{ad}(A)$ acts diagonalizably on $\mathfrak{g}_{0}$. Now $\mathfrak{g}_{0}$ is reductive, so it is the direct sum of the center plus a semisimple subalgebra. The component of $A$ in the center acts trivially under ad, so the semisimple component of $A$ acts diagonalizably under ad, and hence in any finite-dimensional representation. Therefore, $A$ acts diagonalizably in any finite-dimensional representation of $\mathfrak{g}_{0}$ in which the center acts diagonalizably. All representations corresponding to the components of the harmonic curvature 
have this property because they are subquotients of representations of $\mathfrak{g}$ in which the center of $\mathfrak{g}_{0}$ is contained in a Cartan subalgebra. (All real representations of interest we are aware of have this property.)

Definition 2.13. Let $A \in \mathfrak{g}_{0}$, and let $\mathbb{W}$ be a representation of $\mathfrak{g}_{0}$ on which $A$ acts diagonalizably with eigenspace decomposition $\mathbb{W}=$ $\mathbb{W}_{0} \oplus \cdots \oplus \mathbb{W}_{l}$ and eigenvalues $\mu_{i}, i=0, \ldots, l$.

- The stable subspace for $A$, denoted $\mathbb{W}_{s t}(A)$, is the sum of the eigenspaces $\mathbb{W}_{i}$ for which $\mu_{i} \leq 0$.

- The strongly stable subspace for $A$, denoted $\mathbb{W}_{s s}(A)$, is the sum of eigenspaces $\mathbb{W}_{i}$ with $\mu_{i}<0$.

Now our results have the following useful formulation:

Corollary 2.14. In the setting of proposition 2.12, assume that $A=$ $[Z, X] \in \mathfrak{g}_{0}$ and let $\mathbb{W}$ be a representation of $\mathfrak{g}_{0}$. Let $I=(-\epsilon, \epsilon)$.

(1) If $\mathbb{W}_{s t}=\{0\}$, then any $\varphi_{\eta}^{t}$-invariant section of $B \times_{P} \mathbb{W}$ vanishes on $\sigma(I)$.

(2) If $\mathbb{W}_{s s}=\{0\}$, then any $\varphi_{\eta}^{t}$-invariant section of $B \times_{P} \mathbb{W}$ which vanishes at $x_{0}$ vanishes on all of $\sigma(I)$.

(3) Suppose that $\mathbb{W}_{s s}=\{0\}$ and that all eigenvalues of $A$ on $\mathfrak{g}_{-}$are non-positive with the 0-eigenspace equal to $C_{\mathfrak{g}}(Z) \cap \mathfrak{g}_{-}$. Then any $\varphi_{\eta}^{t}$-invariant section of $B \times_{P} \mathbb{W}$ that vanishes at each fixed point of the same geometric type as $x_{0}$ in a neighborhood also vanishes on an open neighborhood of $\sigma(I \backslash\{0\})$ and thus on an open subset containing $x_{0}$ in its closure.

Proof: The $\mu_{i}$-eigenspace $\mathbb{W}_{i}$ for $A$ is the eigenspace for $g(t)=$ $e^{\log (1+s t) A}$ with eigenvalue $\lambda_{i}(t)=(1+s t)^{\mu_{i}}$. Thus $\mathbb{W}_{s t}(A)=\mathbb{W}_{s t}(g)$ and $\mathbb{W}_{s s}(A)=\mathbb{W}_{s s}(g)$ for the holonomy path $g(t)$. After definition 2.10, we observed that if $f: B \rightarrow \mathbb{W}$ is the function corresponding to an invariant section of the bundle $B \times{ }_{P} \mathbb{W}$, then $f(b) \in \mathbb{W}_{s t}(A)$, and if $f\left(b_{0}\right)=0$, then $f(b) \in \mathbb{W}_{s s}(A)$. These facts together with proposition 2.12 yield (1) and (2). 
The additional assumption in (3) implies that for any $Y \in \mathfrak{g}_{-}$, the limit of $\operatorname{Ad}(g(t))(Y)$ as $t \rightarrow \infty$ exists, and equals some $Y_{\infty} \in C_{\mathfrak{g}}(Z) \cap$ $\mathfrak{g}_{-}$. Taking a point $b \in B$ over a point $\sigma(s) \neq x_{0}$ close enough to $x_{0}$, we can thus invoke proposition 2.8 to say that $g(t)$ is a holonomy path at $\exp (b, Y)$ with attractor $\exp \left(b_{0}, Y_{\infty}\right)$ (assuming $Y$ sufficiently close to 0$)$. But $\exp \left(b_{0}, Y_{\infty}\right)$ lies over a higher order fixed point of the same geometric type as $x_{0}$, by proposition 2.5 part (1). Then any $\varphi_{\eta}^{t}$ invariant section must vanish at $\exp (b, Y)$, as well. Varying $Y$ in a neighborhood of 0 in $\mathfrak{g}_{-}$, these points fill a neighborhood of $\sigma(s)$.

The following result strengthens proposition 2.9 in the special case of holonomy paths coming from $\mathfrak{s l}_{2}$-triples. This improvement is crucial, since it provides information on the possible values of invariant sections at the point $b_{0}$ rather than at $b$.

Proposition 2.15. In the setting of proposition 2.12, suppose that $X$ can be chosen so that $A=[Z, X] \in \mathfrak{g}_{0}$. Suppose further that $\mathbb{W}$ is a completely reducible representation of $P$ on which $A$ acts diagonalizably, and that $f: B \rightarrow \mathbb{W}$ is the equivariant function corresponding to $a \varphi_{\eta}^{t}$-invariant section of $B \times_{P} \mathbb{W}$. Then $f\left(b_{0}\right) \in \mathbb{W}_{s t}(A)$.

Proof: Set $b(t)=\exp \left(b_{0}, s X\right) e^{\frac{-t}{1+s t} Z}$ and $b=\exp \left(b_{0}, s X\right) e^{\frac{-1}{s} Z}$, so $g(t)=e^{\log (1+s t) A}$ is a holonomy path at $b$ with attractor $b_{0}$. Let $\mu_{i}$ be the eigenvalues of $A$ on $\mathbb{W}$, so $\lambda_{i}(t)=(1+s t)^{\mu_{i}}$ are the eigenvalues of $g(t)$, for $i=0, \ldots, l$.

Now since $\mathbb{W}$ is a completely reducible representation of $P$, the unipotent radical $P_{+}$acts trivially on $\mathbb{W}$, and for all $t$,

$$
\begin{aligned}
f(b(t)) & =e^{\frac{t}{1+s t} Z} \cdot f\left(\exp \left(b_{0}, s X\right)\right)=f\left(\exp \left(b_{0}, s X\right)\right)=e^{\frac{1}{s} Z} \cdot f\left(\exp \left(b_{0}, s X\right)\right) \\
& =f(b)
\end{aligned}
$$

But then proposition 2.9 (1) simply reads as

$$
\lambda_{i}(t) f(b)_{i} \rightarrow f\left(b_{0}\right)_{i} \quad \text { for } t \rightarrow \infty .
$$

If $\lambda_{i}(t) \rightarrow \infty$, we know that $f(b)_{i}=0$ from proposition 2.9 and thus $f\left(b_{0}\right)_{i}=0$. We conclude $f\left(b_{0}\right) \in \mathbb{W}_{s t}(g)=\mathbb{W}_{s t}(A) . \diamond$ 
Simplifications. The examples we treat below share some features that permit some simplification of these general results and lead to better descriptions of the automorphisms. For $Z \in \mathfrak{p}_{+}$, we don't really use the subsets $F_{\mathfrak{g}}(Z), C_{\mathfrak{g}}(Z)$, and $T_{\mathfrak{g}}(Z)$ of $\mathfrak{g}$, but rather their images in $\mathfrak{g} / \mathfrak{p}$. The improvements are available if one can actually find subsets of $\mathfrak{g}_{-}$that have the same images in $\mathfrak{g} / \mathfrak{p}$. Define $F_{\mathfrak{g}_{-}}(Z)=F_{\mathfrak{g}}(Z) \cap \mathfrak{g}_{-}$, and likewise for $C_{\mathfrak{g}_{-}}$and $T_{\mathfrak{g}_{-}}$.

We give a proof that this simplification is always possible for $|1|$-graded geometries and just verify the facts directly in the other examples. For $|1|$-graded geometries, $\mathfrak{p}_{+}=\mathfrak{g}_{1}$, so $Z \in \mathfrak{p}_{+}$is automatically homogeneous of degree one.

Proposition 2.16. Let $\mathfrak{g}=\mathfrak{g}_{-1} \oplus \mathfrak{g}_{0} \oplus \mathfrak{g}_{1}$ be a $|1|$-graded semsimple Lie algebra and let $Z \in \mathfrak{p}_{+}=\mathfrak{g}_{1}$ be any element.

Any $X \in F_{\mathfrak{g}}(Z)$ is congruent to an element of $F_{\mathfrak{g}_{-}}(Z)$ modulo $\mathfrak{p}$. The analogous statements hold for $C_{\mathfrak{g}}(Z)$ and $T_{\mathfrak{g}}(Z)$. For $X \in T_{\mathfrak{g}_{-}}(Z)$ the commutator $A=[Z, X]$ is always in $\mathfrak{g}_{0}$.

Proof: Decompose $X \in \mathfrak{g}$ as $X=X_{-1}+X_{\mathfrak{p}}$ according to the decomposition $\mathfrak{g}=\mathfrak{g}_{-1} \oplus \mathfrak{p}$. We claim that if $X$ lies in either $F_{\mathfrak{g}}(Z), C_{\mathfrak{g}}(Z)$, or $T_{\mathfrak{g}}(Z)$, then $X_{-1}$ lies in the same subset, which clearly suffices to complete the proof.

First, $[Z, X]=\left[Z, X_{-1}\right]+\left[Z, X_{\mathfrak{p}}\right]$ with the first summand lying in $\mathfrak{g}_{0}$ and the second in $\mathfrak{g}_{1}$. This already implies the claim for $C_{\mathfrak{g}}(Z)$. Next, $[[Z, X], X]$ is congruent to $\left[\left[Z, X_{-1}\right], X_{-1}\right] \in \mathfrak{g}_{-1}$ modulo $\mathfrak{p}$. Hence if $X \in F_{\mathfrak{g}}(Z)$, then we must have $\left[\left[Z, X_{-1}\right], X_{-1}\right]=0$. Since the condition $\operatorname{ad}\left(X_{-1}\right)(Z) \in \mathfrak{p}$ always holds, and $\operatorname{ad}\left(X_{-1}\right)^{k}(Z)=0$ for all $k \geq 3$, we see that $X_{-1} \in F_{\mathfrak{g}}(Z)$, which completes the proof for this subset.

Since $[[Z, X], X]$ is congruent to $\left[\left[Z, X_{-1}\right], X_{-1}\right] \in \mathfrak{g}_{-1}$ modulo $\mathfrak{p}$, we also see that if $X \in T_{\mathfrak{g}}(Z)$ then $\left[\left[Z, X_{-1}\right], X_{-1}\right]=-2 X_{-1}$. But since $\mathfrak{g}_{1}$ is abelian, we also get $[[Z, X], Z]=\left[\left[Z, X_{-1}\right], Z\right]$, which completes the proof. $\diamond$

Next, there are obvious improvements of our basic results, propositions 2.5 and 2.12 , in the case that one can find such nicer representatives. 
Suppose we have given a Cartan geometry $(M, B, \omega)$ of type $(\mathfrak{g}, P)$ and $\eta \in \mathfrak{i n f}(M)$ with a higher order fixed point at $x_{0} \in M$ with isotropy $\alpha \in T_{x_{0}}^{*}(M)$. Suppose that for $\xi \in F(\alpha)$, there is $b_{0} \in \pi^{-1}\left(x_{0}\right)$ that identifies $\xi$ with some $X \in F_{\mathfrak{g}_{-}}(Z)$, where $Z \in \mathfrak{p}_{+}$is the isotropy of $\eta$ with respect to $b_{0}$. Then the curve $r \mapsto \exp \left(b_{0}, r X\right)$ in part (1) of proposition 2.5 is a distinguished curve of the geometry. If $\xi \in C(\alpha)$ and we can find a representative $X \in C_{\mathfrak{g}_{-}}(Z)$, then one again obtains a distinguished curve in part (1) of proposition 2.5.

Finally, if $\xi \in T(\alpha)$ and we find a representative $X \in T_{\mathfrak{g}_{-}}(Z)$ then the curve $\sigma$ constructed in the proposition 2.12 again is a distinguished curve.

We obtain the following formulation of these simplifications for $|1|-$ graded geometries using the normal coordinates defined in section 1.1.2.

Proposition 2.17. Consider a Cartan geometry $(M, B, \omega)$ of type $(\mathfrak{g}, P)$ corresponding to a $|1|$-grading $\mathfrak{g}=\mathfrak{g}_{-1} \oplus \mathfrak{g}_{0} \oplus \mathfrak{g}_{1}$. Let $\eta \in \mathfrak{i n} \mathfrak{f}(M)$ have a higher order fixed point at $x_{0} \in M$ with isotropy $\alpha \in T_{x_{0}}^{*}(M)$.

Then in any normal coordinate chart centered at $x_{0}$, the subset $F(\alpha)$ consists of fixed points and $C(\alpha) \subset F(\alpha)$ consists of higher order fixed points of the same geometric type as $x_{0}$. If this chart has image $U \subset$ $T_{x_{0}} M$, the action of the local flow $\varphi_{\eta}^{t}$ on $U \cap \mathbf{R}^{*} T(\alpha)$ is given by $\lambda \xi \mapsto$ $\frac{\lambda}{1+\lambda t} \xi$ for $\xi \in T(\alpha)$ and $\lambda t>0$.

Proof: Given any $b_{0} \in \pi^{-1}\left(x_{0}\right)$ we let $Z \in \mathfrak{g}_{1}$ be the element corresponding to $\alpha$ via $b_{0}$. Then by proposition 2.16 there is a representative $X \in \mathfrak{g}_{-}$for any $\xi$ in each of the subsets $C(\alpha), F(\alpha)$, and $T(\alpha)$. Then the curves $\exp \left(b_{0}, \lambda X\right)$ used in the proofs of propositions 2.5 (3) and 2.12 are the images of the line spanned by $X$ under the inverse of the normal coordinate chart defined at $b_{0}$. Hence the claim follows from the two propositions. $\diamond$

\section{Applications}


3.1. Almost-Grassmannian structures of type $(2, n)$. The homogeneous model for almost-Grassmannian structures is the Grassmannian $\operatorname{Gr}(m, m+n)$, viewed as a homogeneous space $\mathrm{SL}(m+n, \mathbf{R}) / P$, where $P$ is the parabolic subgroup preserving an $m$-dimensional subspace of $\mathbf{R}^{m+n}$. A normal Cartan geometry of type $(\operatorname{SL}(m+n), P)$ on a manifold $M$ of dimension $m n$ is equivalent to a first order $\mathrm{G}$-structure of type $G_{0}=S(\mathrm{GL}(m, \mathbf{R}) \times G L(n, \mathbf{R}))<G L(m+n, \mathbf{R})$. Here $G_{0}$ acts on the space $\mathbf{R}^{n \times m}$ of $n \times m$-matrices by multiplications from both sides. Explicitly, such a reduction of structure group can be described by the following data:

- auxiliary vector bundles $E$ and $F$ over $M$, with fibers $\mathbf{R}^{m}$ and $\mathbf{R}^{n}$, respectively

- an isomorphism $T M \cong E^{*} \otimes F$

- a trivialization $\wedge^{m} E^{*} \otimes \wedge^{n} F \cong M \times \mathbf{R}$

More details on almost-Grassmannian structures and their description as parabolic geometries can be found in section 4.1.3 of [4]. There are two harmonic curvature components, which will be described in some detail below. One is a torsion, the vanishing of which is equivalent to the Cartan geometry being torsion-free. The other harmonic component is a curvature, with values in $\mathfrak{g}_{0}$.

The cotangent bundle $T^{*} M$ can be identified with $F^{*} \otimes E$ by $\alpha(v)=$ $\operatorname{tr}_{E}(\alpha \circ v)=\operatorname{tr}_{F}(v \circ \alpha)$. It is associated to the $G_{0}$ representation on $\mathbf{R}^{m \times n}$. The geometric types of cotangent vectors are given by the ranks of the corresponding matrices. Here we will consider almostGrassmannian structures of type $(2, n)$ for $n \geq 2$, so the possible ranks of non-zero cotangent vectors are 1 and 2 , and we will in particular prove the following result:

Theorem 3.1. Let $M$ be endowed with an almost-Grassmannian structure of type $(2, n), n \geq 2$, and let $\eta \in \mathfrak{i n f} \mathfrak{f}(M)$ have a higher order fixed point at $x_{0}$.

(1) Higher order fixed points with isotropy of rank two are smoothly isolated in the strongly fixed set. For rank one isotropy, the 
strongly fixed component of $x_{0}$ contains a submanifold of $d i$ mension $n-1$.

(2) If the isotropy of $\eta$ at $x_{0}$ has rank two, or if the geometry is torsion free, then there is an open set $U \subset M$ with $x_{0} \in \bar{U}$ on which the almost-Grassmannian structure is locally flat.

This result will be a consequence of propositions 3.3 and 3.5 below, which give a more detailed description of the flow for each of the two possible geometric types.

The Lie algebra $\mathfrak{s l}(n+2, \mathbf{R})$ has a $|1|$-grading coming from the block decomposition

$$
\left(\begin{array}{cc}
\mathfrak{g}_{0} & \mathfrak{g}_{1} \\
\mathfrak{g}_{-1} & \mathfrak{g}_{0}
\end{array}\right)
$$

with block sizes 2 and $n$. Here $\mathfrak{g}_{0}=\mathfrak{s}(\mathfrak{g l}(2, \mathbf{R}) \times \mathfrak{g l}(n, \mathbf{R}))$ and, as $\mathfrak{g}_{0}$-modules, we have $\mathfrak{g}_{-1}=L\left(\mathbf{R}^{2}, \mathbf{R}^{n}\right)$ and $\mathfrak{g}_{1}=L\left(\mathbf{R}^{n}, \mathbf{R}^{2}\right)$. The dual pairing between $\mathfrak{g}_{-1}$ and $\mathfrak{g}_{1}$ is given by $(X, Z) \mapsto \operatorname{tr}(Z X)=\operatorname{tr}(X Z)$, while the bracket of these two is $[Z, X]=(Z X,-X Z) \in \mathfrak{g}_{0}$. Further,

$$
[[Z, X], X]=-2 X Z X \quad \text { and } \quad[[Z, X], Z]=2 Z X Z
$$

In terms of the standard representations $\mathbf{R}^{2}$ and $\mathbf{R}^{n}$ of the two factors of $\mathfrak{g}_{0}$, we have $\mathfrak{g}_{-1} \cong \mathbf{R}^{2 *} \otimes \mathbf{R}^{n}$ and $\mathfrak{g}_{1} \cong \mathbf{R}^{2} \otimes \mathbf{R}^{n *}$; consequently,

$$
\Lambda^{2} \mathfrak{g}_{1}=\left(\Lambda^{2} \mathbf{R}^{2} \otimes S^{2} \mathbf{R}^{n *}\right) \oplus\left(S^{2} \mathbf{R}^{2} \otimes \Lambda^{2} \mathbf{R}^{n *}\right) .
$$

We will apply the general results of section 2 to the harmonic curvatures, which, for $n \geq 3$, are the highest weight components

$$
\mathbb{V} \subset\left(S^{2} \mathbf{R}^{2} \otimes \Lambda^{2} \mathbf{R}^{n *}\right) \otimes\left(\mathbf{R}^{2 *} \otimes \mathbf{R}^{n}\right) \subset \Lambda^{2} \mathfrak{g}_{1} \otimes \mathfrak{g}_{-1},
$$

and

$$
\mathbb{U} \subset\left(\Lambda^{2} \mathbf{R}^{2} \otimes S^{2} \mathbf{R}^{n *}\right) \otimes \mathfrak{s l}(n, \mathbf{R}) \subset \Lambda^{2} \mathfrak{g}_{1} \otimes \mathfrak{g}_{0}
$$

The first part will be called the "harmonic torsion," denoted $\tau$ below, and the second the "harmonic curvature," denoted $\rho$. By the general theory, vanishing of the harmonic torsion is equivalent to torsion freeness, while vanishing of both the harmonic curvature and the harmonic torsion on an open subset is equivalent to local flatness. We will write $\mathbb{V}^{1}=S^{2} \mathbf{R}^{2} \otimes \mathbf{R}^{2 *}$ and $\mathbb{V}^{2}=\Lambda^{2} \mathbf{R}^{n *} \otimes \mathbf{R}^{n}$ below, so $\mathbb{V} \subset \mathbb{V}^{1} \otimes \mathbb{V}^{2}$. 
Almost-Grassmannian structures of type $(2,2)$ are equivalent to 4dimensional conformal spin structures in split signature $(2,2)$ via the isomorphisms $G=\operatorname{SL}(4, \mathbf{R}) \cong \operatorname{Spin}(3,3)$ and $G_{0}=S(\mathrm{GL}(2, \mathbf{R}) \times$ $\mathrm{GL}(2, \mathbf{R})) \cong \operatorname{CSpin}(2,2)$. The tangent bundle naturally can be written as the tensor product of the two basic real spinor bundles on 4-dimensional conformal manifolds of split signature. Almost Grassmannian structures of type $(2, n)$ provide a natural higher dimensional analog of this so-called spinor formalism in 4-dimensional conformal geometry. For type $(2,2)$, the harmonic curvature still consists of two components, but there are two curvatures instead of one torsion and one curvature, the self-dual and anti-self-dual parts of the Weyl curvature. Both curvatures have values in bundles associated to a highest weight subspace in $\left(\Lambda^{2} \mathbf{R}^{2} \otimes S^{2} \mathbf{R}^{2 *}\right) \otimes \mathfrak{s l}(2, \mathbf{R})$, of which there are two. We will assume $n \geq 3$ below, but our arguments also apply to the case $n=2$, and thus provide another proof for split signature conformal structures in dimension 4.

3.1.1. The rank two case. We start by collecting the algebraic results.

Lemma 3.2. Suppose that $Z \in L\left(\mathbf{R}^{n}, \mathbf{R}^{2}\right) \cong \mathfrak{g}_{1}$ has rank two. Then

(1) The subspaces associated to $Z$ are

$$
\begin{aligned}
\{0\}= & C_{\mathfrak{g}_{-}}(Z) \subset F_{\mathfrak{g}_{-}}(Z)=\left\{X \in L\left(\mathbf{R}^{2}, \mathbf{R}^{n}\right): X Z X=0\right\} \\
& T_{\mathfrak{g}_{-}}(Z)=\left\{X \in L\left(\mathbf{R}^{2}, \mathbf{R}^{n}\right): Z X=\operatorname{Id}_{\mathbf{R}^{2}}\right\} \\
\cong & \left\{W \subset \mathbf{R}^{n}: \operatorname{dim} W=2, W \cap \operatorname{ker}(Z)=\{0\}\right\}
\end{aligned}
$$

where the last isomorphism is induced by $X \mapsto \operatorname{im}(X)$.

(2) For $X \in T_{\mathfrak{g}_{-}}(Z)$, let $A=[Z, X]$ and $W=\operatorname{im}(X)$. Then the eigenvalues of $A$ on $\mathfrak{g}_{-1}$ are all negative. For the representation $\mathbb{V}$ corresponding to the harmonic torsion, $\mathbb{V}_{s s}(A)=0$, and

$$
\mathbb{V}_{s t}(A) \subset\left(S^{2} \mathbf{R}^{2} \otimes \Lambda^{2} \mathbf{R}^{n *}\right) \otimes\left(\mathbf{R}^{2 *} \otimes W\right)
$$

For the representation $\mathbb{U}$ corresponding to the harmonic curvature, $\mathbb{U}_{s t}(A)=\{0\}$. 
Proof: By assumption, $Z$ is onto and $\operatorname{ker}(Z) \subset \mathbf{R}^{n}$ is a subspace of dimension $n-2$. From the brackets computed above, the descriptions of $C_{\mathfrak{g}_{-}}(Z)$ and $F_{\mathfrak{g}_{-}}(Z)$ follow immediately.

For $X \in L\left(\mathbf{R}^{2}, \mathbf{R}^{n}\right)$, the condition $[[Z, X], Z]=2 Z$ implies $Z X Z=Z$, so any $X \in T_{\mathfrak{g}_{-}}(Z)$ has rank two and $\operatorname{im}(X) \cap \operatorname{ker}(Z)=\{0\}$. For any two-dimensional subspace $W \subset \mathbf{R}^{n}$ complementary to $\operatorname{ker}(Z)$, the map $Z$ restricts to a linear isomorphism $W \rightarrow \mathbf{R}^{2}$; if $\operatorname{im}(X)=W$, then $Z X Z=Z$ if and only if $X$ is the inverse of this isomorphism. In this case, $Z X=\operatorname{Id}_{\mathbf{R}^{2}}$ and $[[Z, X], X]=-2 X$ follows automatically. Hence

$$
T_{\mathfrak{g}_{-}}(Z)=\left\{W \subset \mathbf{R}^{n}: \operatorname{dim} W=2, W \cap \operatorname{ker} Z=\{0\}\right\}
$$

via $X=\left(\left.Z\right|_{W}\right)^{-1}$. We also see immediately that $A=[Z, X]$ acts by 1 on $\mathbf{R}^{2}$, while on $\mathbf{R}^{n}$, the eigenspaces are $W$ and $\operatorname{ker}(Z)$ with eigenvalues -1 and 0 , respectively. Thus all eigenvalues of $A$ on $\mathfrak{g}_{-} \cong L\left(\mathbf{R}^{2}, \mathbf{R}^{n}\right)$ are negative.

To analyze the representation

$$
\left(S^{2} \mathbf{R}^{2} \otimes \Lambda^{2} \mathbf{R}^{n *}\right) \otimes\left(\mathbf{R}^{2 *} \otimes \mathbf{R}^{n}\right),
$$

observe that $A$ acts as the identity on $S^{2} \mathbf{R}^{2} \otimes \mathbf{R}^{2 *}$. The eigenspaces in $\mathbf{R}^{n *}$ are $W^{o}$ and $\operatorname{ker}(Z)^{o}$, the respective annihilators of $W$ and $\operatorname{ker}(Z)$, with eigenvalues 0 and 1 , respectively. Hence the eigenspace structure on $\Lambda^{2} \mathbf{R}^{n *} \otimes \mathbf{R}^{n}$ is:

\begin{tabular}{|c|c|}
\hline subspace & eigenvalue \\
\hline$\Lambda^{2} \operatorname{ker}(Z)^{o} \otimes \operatorname{ker}(Z)$ & 2 \\
$\Lambda^{2} \operatorname{ker}(Z)^{o} \otimes W \oplus\left(\operatorname{ker}(Z)^{o} \wedge W^{o}\right) \otimes \operatorname{ker}(Z)$ & 1 \\
$\left(\operatorname{ker}(Z)^{o} \wedge W^{o}\right) \otimes W \oplus \Lambda^{2} W^{o} \otimes \operatorname{ker}(Z)$ & 0 \\
$\Lambda^{2} W^{o} \otimes W$ & -1 \\
\hline
\end{tabular}

The only nonnegative eigenvalue on $\mathbb{V}$ is 0 , and the claim $\mathbb{V}_{s t}=S^{2} \mathbf{R}^{2} \otimes$ $\Lambda^{2} W^{o} \otimes \mathbf{R}^{2 *} \otimes W$ follows.

Next consider

$$
\left(\Lambda^{2} \mathbf{R}^{2} \otimes S^{2} \mathbf{R}^{n *}\right) \otimes \mathfrak{s l}(n, \mathbf{R}) .
$$

Here $A$ acts by multiplication by 2 on $\Lambda^{2} \mathbf{R}^{2}$, while on $S^{2} \mathbf{R}^{n *}$ the possible eigenvalues are 0,1 , and 2. Finally, on $\mathfrak{s l}(n, \mathbf{R})$ the possible 
eigenvalues evidently are $-1,0$, and 1 . Thus all eigenvalues on $\mathbb{U}$ are positive. $\diamond$

These results can be immediately translated to geometry:

Proposition 3.3. Let $M$ be endowed with an almost-Grassmannian structure of type $(2, n), n \geq 2$, and let $\eta \in \mathfrak{i n f}(M)$ vanish to higher order at $x_{0} \in M$. Assume that the isotropy of $\eta$ is $\alpha \in T_{x_{0}}^{*} M \cong L\left(F_{x_{0}}, E_{x_{0}}\right)$ of rank two.

(1) The subsets $C(\alpha) \subset F(\alpha)$ of $T_{x_{0}} M \cong L\left(F_{x_{0}}, E_{x_{0}}\right)$ are

$$
\{0\}=C(\alpha) \subset F(\alpha)=\{\xi: \xi \circ \alpha \circ \xi=0\}
$$

and

$$
T(\alpha)=\left\{\xi: \alpha \circ \xi=\operatorname{Id}_{E_{x_{0}}}\right\}
$$

(2) In any normal coordinate chart centered at $x_{0}$, there is an open neighborhood $U$ of 0 such that

(a) The higher order fixed point $x_{0}$ is smoothly isolated in the strongly fixed set of the flow, and elements of $F(\alpha) \cap U$ are fixed points.

(b) For any $\xi \in U$ in the cone

$$
S=\left\{\xi: \alpha \circ \xi=\lambda \operatorname{Id}_{F_{x_{0}}} \text { for some } \lambda \neq 0\right\}
$$

the action of the flow in normal coordinates is

$$
\varphi_{\eta}^{t}(\xi)=\frac{1}{2+t \cdot \operatorname{tr}(\alpha \circ \xi)} \cdot \xi \quad \text { for } t \cdot \operatorname{tr}(\alpha \circ \xi)>0
$$

(3) There is an open neighborhood of $S \backslash\{0\}$ on which the geometry is locally flat.

Proof: From lemma 3.2, it is clear that $\xi \in T_{x_{0}} M$ lies in $F(\alpha)$ if and only if $\xi \circ \alpha \circ \xi=0$. On the other hand, $\xi$ is a multiple of an element $\xi_{0} \in T(\alpha)$ if and only if $\alpha \circ \xi$ is a multiple of the identity, and then $\xi=\frac{\operatorname{tr}(\alpha \circ \xi)}{2} \cdot \xi_{0}$. Using this, (1) and (2) follow directly from propositions 2.5 and 2.17 .

To prove part (3), let $\xi \in T(\alpha)$, and apply proposition 2.12 to the line $l$ spanned by $\xi$. Now corollary 2.14 together with lemma 3.2 shows that 
the harmonic curvature $\rho$ vanishes on a neighborhood of $l \backslash\{0\}$. Thus this curvature vanishes on a neighborhood of $S \backslash\{0\}$.

For the harmonic torsion, apply proposition 2.15 to the description of $\mathbb{V}_{s s}(A)$ in lemma 3.2 to see that for any $u, v \in T_{x_{0}} M$, the harmonic torsion $\tau_{x_{0}}(u, v) \in T_{x_{0}} M \cong L\left(E_{x_{0}}, F_{x_{0}}\right)$ has values in $\operatorname{im}(\xi) \subset F_{x_{0}}$. The same holds for any $\xi$; from lemma 3.2 , im $(\xi)$ can be any twodimensional subspace complementary to $\operatorname{ker}(\alpha) \subset F_{x_{0}}$. The intersection of all such subspaces is 0 , so $\tau\left(x_{0}\right)=0$. Now corollary 2.14 (3) implies that $\tau$ vanishes on an open neighborhood of $S \backslash\{0\}$. Thus all harmonic curvature components vanish locally around $S \backslash\{0\}$, so the geometry is flat on an open set as claimed. $\diamond$

\subsubsection{The rank one case.}

Lemma 3.4. Let $Z \in L\left(\mathbf{R}^{n}, \mathbf{R}^{2}\right)=\mathfrak{g}_{1}$ be of rank one.

(1) The sets associated to $Z$ are

$$
\begin{gathered}
C_{\mathfrak{g}_{-}}(Z)=\left\{X \in L\left(\mathbf{R}^{2}, \mathbf{R}^{n}\right): \operatorname{im}(Z) \subset \operatorname{ker}(X) \text { and } \operatorname{im}(X) \subset \operatorname{ker}(Z)\right\} \\
F_{\mathfrak{g}_{-}}(Z)=\left\{X \in L\left(\mathbf{R}^{2}, \mathbf{R}^{n}\right): X Z X=0\right\} \\
T_{\mathfrak{g}_{-}}(Z)=\left\{X \in L\left(\mathbf{R}^{2}, \mathbf{R}^{n}\right): \operatorname{rk}(X)=1, \operatorname{tr}(Z X)=\operatorname{tr}(X Z)=1\right\}
\end{gathered}
$$

(2) For any choice of lines $V \subset \mathbf{R}^{2}$ transversal to $\operatorname{im}(Z)$ and $W \subset$ $\mathbf{R}^{n}$ transversal to $\operatorname{ker}(Z)$, there is a unique element $X \in T_{\mathfrak{g}_{-}}(Z)$ with $\operatorname{ker}(X)=V$ and $\operatorname{im}(X)=W$.

(3) Let $X, V$, and $W$ be as in (2). Let $V^{\circ} \subset \mathbf{R}^{2 *}$ and $W^{\circ} \subset \mathbf{R}^{n *}$ be the annihlators, and let $A=[Z, X]$. Then all eigenvalues of $A$ on $\mathfrak{g}_{-1}$ are non-positive, and the 0-eigenspace coincides with $C_{\mathfrak{g}_{-}}(Z)$. Moreover, for the representations $\mathbb{V} \subset \mathbb{V}^{1} \otimes \mathbb{V}^{2}$ corresponding to the harmonic torsion and $\mathbb{U}$ corresponding to the harmonic curvature,
(a) $\mathbb{V}_{s s}^{1}(A)=S^{2} V \otimes V^{\circ}$
(b) $\mathbb{V}_{s t}^{1}(A) \subset S^{2} V \otimes \mathbf{R}^{2 *}+\left(V \odot \mathbf{R}^{2}\right) \otimes V^{\circ}$
(c) $\mathbb{V}_{s s}^{2}(A)=\Lambda^{2} W^{\circ} \otimes W$
(d) $\mathbb{V}_{s t}^{2}(A) \subset \Lambda^{2} W^{\circ} \otimes \mathbf{R}^{n}+\left(W^{\circ} \wedge \mathbf{R}^{n *}\right) \otimes W$
(e) $\mathbb{V}_{s s}(A) \subset \mathbb{V}_{s s}^{1}(A) \otimes \mathbb{V}_{s t}^{2}(A)+\mathbb{V}_{s t}^{1}(A) \otimes \mathbb{V}_{s s}^{2}(A)$ 
(f) $\mathbb{V}_{s t}(A) \cap\left(\left(S^{2} \mathbf{R}^{2} \otimes \Lambda^{2} \mathbf{R}^{n *}\right) \otimes C_{\mathfrak{g}_{-}}(Z)\right)$ $\subset\left(S^{2} V \otimes \Lambda^{2} W^{\circ}\right) \otimes C_{\mathfrak{g}_{-}}(Z)$

(g) $\mathbb{U}_{s s}(A)=\{0\}$

(h) $\mathbb{U}_{s t}(A) \subset\left(\Lambda^{2} \mathbf{R}^{2} \otimes S^{2} \mathbf{R}^{n *}\right) \otimes \mathbf{R}^{n *} \otimes W$

Proof: (1) Since $Z$ has rank one, $\operatorname{ker}(Z) \subset \mathbf{R}^{n}$ has dimension $n-1$ and $\operatorname{im}(Z) \subset \mathbf{R}^{2}$ is one-dimensional. Now $X \in \mathfrak{g}_{-1} \cong L\left(\mathbf{R}^{2}, \mathbf{R}^{n}\right)$ lies in $C_{\mathfrak{g}_{-}}(Z)$ if and only if $Z X=0$ and $X Z=0$, that is, if and only if $\operatorname{im}(Z) \subset \operatorname{ker}(X)$ and $\operatorname{im}(X) \subset \operatorname{ker}(Z)$. For $X \neq 0$, this means $\operatorname{ker}(X)=\operatorname{im}(Z)$, so $X$ has rank one, and we obtain the description of $C_{\mathfrak{g}_{-}}(Z)$. The description of $F_{\mathfrak{g}_{-}}(Z)=\{X: X Z X=0\}$ follows exactly as in the proof of lemma 3.2 .

Next, $X \in T_{\mathfrak{g}_{-}}(Z)$ is evidently equivalent to $X Z X=X$ and $Z X Z=Z$. Since $\operatorname{rk}(X Z X) \leq 1$, the first equality implies $\operatorname{rk}(X)=1$. Also, $(Z X)^{2}=Z X$, so $Z X$ is a rank one projection with $\operatorname{tr}(Z X)=1$. Conversely, suppose $\operatorname{rk}(X)=1$ and $\operatorname{tr}(Z X)=1$. Then $\operatorname{im}(X)$ is transversal to $\operatorname{ker}(Z)$ and $\operatorname{im}(Z)$ is transversal to $\operatorname{ker}(X)$. Taking a basis adapted to the splitting $\mathbf{R}^{2}=\operatorname{ker}(X) \oplus \operatorname{im}(Z)$, we conclude that $Z X$ is the projection onto the second factor, so $Z X Z=Z$ and $X Z X=X$. Now (1) is proved.

(2) Given lines $W$ and $V$ as in the statement, there is a unique linear map $X: \mathbf{R}^{2} \rightarrow \mathbf{R}^{n}$ with $\operatorname{ker}(X)=V$ and $\operatorname{im}(X)=W$ up to scale. Now $Z$ induces a linear isomorphism $W \rightarrow \mathbf{R}^{2} / V$, and the remaining condition $\operatorname{tr}(Z X)=1$ for $X \in T_{\mathfrak{g}_{-}}(Z)$ is equivalent to $X$ inducing the inverse of this isomorphism.

(3) Let $X$ be as in (2), so $Z X$ is the projection onto the first factor of $\mathbf{R}^{2}=\operatorname{im}(Z) \oplus V$, while $X Z$ is the second projection in $\mathbf{R}^{n}=\operatorname{ker}(Z) \oplus$ $W$. Hence $A=[Z, X]$ has eigenvalues 1 on $\operatorname{im}(Z)$ and 0 on $V=$ $\operatorname{ker}(X)$, and eigenvalues 0 on $\operatorname{ker}(Z)$ and -1 on $W$. The eigenspace decompositions $\mathbf{R}^{n *}=W^{\circ} \oplus \operatorname{ker}(Z)^{\circ}$, with respective eigenvalues 0 and 1 , and $\mathbf{R}^{2 *}=V^{\circ} \oplus \operatorname{im}(Z)^{\circ}$, with respective eigenvalues -1 and 0 , follow, which implies all our claims on eigenvalues and eigenspaces for $\mathfrak{g}_{-1} \cong L\left(\mathbf{R}^{2}, \mathbf{R}^{n}\right)$. 
Next, it is easy to compute the eigenspace decomposition of $\mathbb{V}^{1} \cong$ $S^{2} \mathbf{R}^{2} \otimes \mathbf{R}^{2 *}$.

\begin{tabular}{|c|c|}
\hline subspace & eigenvalue \\
\hline$S^{2}(\operatorname{im}(Z)) \otimes \operatorname{im}(Z)^{o}$ & 2 \\
$S^{2}(\operatorname{im}(Z)) \otimes V^{o} \oplus(\operatorname{im}(Z) \odot V) \otimes \operatorname{im}(Z)^{o}$ & 1 \\
$(\operatorname{im}(Z) \odot V) \otimes V^{o} \oplus S^{2}(V) \otimes \operatorname{im}(Z)^{o}$ & 0 \\
$S^{2}(V) \otimes V^{o}$ & -1 \\
\hline
\end{tabular}

and of $\mathbb{V}^{2} \cong \Lambda^{2} \mathbf{R}^{n *} \otimes \mathbf{R}^{n}$ :

\begin{tabular}{|c|c|}
\hline subspace & eigenvalue \\
\hline$\Lambda^{2} \operatorname{ker}(Z)^{o} \otimes \operatorname{ker}(Z)$ & 2 \\
$\Lambda^{2} \operatorname{ker}(Z)^{o} \otimes W \oplus\left(\operatorname{ker}(Z)^{o} \wedge W^{o}\right) \otimes \operatorname{ker}(Z)$ & 1 \\
$\left(\operatorname{ker}(Z)^{o} \wedge W^{o}\right) \otimes W \oplus \Lambda^{2} W^{o} \otimes \operatorname{ker}(Z)$ & 0 \\
$\Lambda^{2} W^{o} \otimes W$ & -1 \\
\hline
\end{tabular}

Now the claims $(\mathrm{a})-(\mathrm{d})$ on $\mathbb{V}_{s s}^{i}(A)$ and $\mathbb{V}_{s t}^{i}(A)$, for $i=1,2$, can be read from the tables.

To get a negative eigenvalue on $\mathbb{V}^{1} \otimes \mathbb{V}^{2}$ one has to have eigenvalue -1 on one factor and a non-positive eigenvalue on the other, which proves the claim (e) on $\mathbb{V}_{s s}(A)$. Since $C_{\mathfrak{g}_{-}}(Z)$ is the zero eigenspace for $A$ in $\mathfrak{g}_{-1}$, and all eigenvalues on $S^{2} \mathbf{R}^{2}$ and $\Lambda^{2} \mathbf{R}^{n *}$ are non-negative, a non-positive eigenvalue on $\left(S^{2} \mathbf{R}^{2} \otimes \Lambda^{2} \mathbf{R}^{n *}\right) \otimes C_{\mathfrak{g}_{-}}(Z)$ is only possible if the eigenvalue on the first factor is zero. This implies the claim (f) on $\mathbb{V}_{s t}(A)$.

To deal with $\mathbb{U}$, observe that on $\Lambda^{2} \mathbf{R}^{2}, A$ acts as the identity, while the possible eigenvalues on $S^{2} \mathbf{R}^{n *}$ range between 0 and 2 . On $\mathfrak{s l}(n, \mathbf{R})$ the eigenspace decomposition is

\begin{tabular}{|c|c|}
\hline subspace & eigenvalue \\
\hline$W^{o} \otimes W$ & -1 \\
$\operatorname{ker}(Z)^{o} \otimes W \oplus W^{o} \otimes \operatorname{ker}(Z)$ & 0 \\
$\operatorname{ker}(Z)^{o} \otimes \operatorname{ker}(Z)$ & 1 \\
\hline
\end{tabular}

The (-1)-eigenspace consists of maps having values in $W$, so the claims (g) and (h) that $\mathbb{U}_{s s}(A)=0$ and $\mathbb{U}_{s t}(A) \subset\left(\Lambda^{2} \mathbf{R}^{2} \otimes S^{2} \mathbf{R}^{n *}\right) \otimes \mathbf{R}^{n *} \otimes W$ are proved. $\diamond$ 
A linear map $X: \mathbf{R}^{2} \rightarrow \mathbf{R}^{n}$ of rank one with $\operatorname{ker}(X)=V$ and $\operatorname{im}(X)=W$ naturally determines an $(n+1)$-dimensional subspace $\mathfrak{a}(X) \subset L\left(\mathbf{R}^{2}, \mathbf{R}^{n}\right)$, where

$$
X \in \mathfrak{a}(X)=\{Y: \operatorname{im}(Y) \subseteq W\}+\{Y: V \subseteq \operatorname{ker}(Y)\}
$$

The intersection of the two summands is the line spanned by $X$. For $\xi \in$ $T_{x} M$ of rank one, denote by $\mathfrak{a}(\xi) \subseteq T_{x} M$ the corresponding subspace.

Proposition 3.5. Let $M$ be endowed with an almost-Grassmannian structure of type $(2, n), n \geq 2$, and let $\eta \in \mathfrak{i n f}(M)$ vanish to higher order at $x_{0} \in M$. Assume that the isotropy $\alpha \in T_{x_{0}}^{*} M \cong L\left(F_{x_{0}}, E_{x_{0}}\right)$ of $\eta$ has rank one.

(1) The subsets $C(\alpha) \subset F(\alpha)$ of $T_{x_{0}} M \cong L\left(E_{x_{0}}, F_{x_{0}}\right)$ are given by $\{\xi: \alpha \circ \xi=\xi \circ \alpha=0\}=C(\alpha) \subset F(\alpha)=\{\xi: \xi \circ \alpha \circ \xi=0\}$.

Moreover, for each choice of lines $V \subset E_{x_{0}}$ transversal to $\operatorname{im}(\alpha)$ and $W \subset F_{x_{0}}$ transversal to $\operatorname{ker}(\alpha)$, there is a unique element $\xi \in T(\alpha)$ with $\operatorname{ker}(\xi)=V$ and $\operatorname{im}(\xi)=W$.

(2) For any normal coordinate chart centered at $x_{0}$, there is an open neighborhood $U$ of 0 such that

(a) Elements of $F(\alpha) \cap U$ are fixed points and $C(\alpha) \cap U$ lies in the strongly fixed component of $x_{0}$.

(b) For any rank one element $\xi \in U$ such that $\alpha(\xi) \neq 0$, the flow acts in normal coordinates by $\varphi_{\eta}^{t}(\xi)=\frac{1}{1+t \alpha(\xi)} \cdot \xi$, whenever $\operatorname{t} \alpha(\xi)>0$.

(3) Both the harmonic torsion $\tau$ and the harmonic curvature $\rho$ vanish on $C(\alpha) \cap U$.

(4) Let $\xi$ be as in (2)(b) above, and let $c$ be the distinguished curve obtained from the line spanned by $\xi$. Then

(a) There is a neighborhood $U_{0}$ of $c \backslash\left\{x_{0}\right\}$ in $M$ on which the harmonic curvature $\rho$ vanishes identically. In particular, if the geometry is torsion free, then it is flat on $U_{0}$.

(b) The harmonic torsion $\tau \in \Omega^{2}(M, T M)$ has the following properties along $c \backslash\left\{x_{0}\right\}$ : 
(i) $i_{\eta}(\tau)=0$, and $\tau_{x}(u, v) \in \mathfrak{a}(\eta(x))$ for any $u, v \in T_{x} M$.

(ii) If $\xi \in \mathfrak{a}(\eta(x))$, then $i_{\xi} \tau_{x}$ vanishes on $\mathfrak{a}(\eta(x))$ and has values in $\mathbf{R} \eta(x)$.

Moreover, $\tau$ has the same algebraic type on a neighborhood of $c \backslash\left\{x_{0}\right\}$.

Proof: Point (1) immediately follows from lemma 3.4. Then part (a) of (2) follows from proposition 2.17. The elements $\xi$ in part (b) of (2) are exactly the positive multiples of elements $\xi_{0} \in T(\alpha)$, and $\xi=\alpha(\xi) \xi_{0}$. Thus (2)(b) also follows from proposition 2.17 .

For part (3), first consider the harmonic torsion and curvature at the point $x_{0}$. Let $\xi \in T(\alpha)$. By proposition 2.15 and lemma 3.4, $\rho_{x_{0}}(u, v)$, for any $u, v \in T_{x_{0}} M$, is an endomorphism of $F_{x_{0}}$ with values $\operatorname{in} \operatorname{im}(\xi)$. Varying $\xi \in T(\alpha)$ gives all lines transversal to $\operatorname{ker}(\alpha)$, so $\rho_{x_{0}}=0$.

To show that $\tau\left(x_{0}\right)=0$, use the observation in section 2.3 of [5] that for the value $\kappa\left(b_{0}\right) \in \Lambda^{2} \mathfrak{g}_{1} \otimes \mathfrak{g}$ of the curvature function and for $Z \in \mathfrak{g}_{1}$ corresponding to $\eta\left(x_{0}\right)$ via $b_{0}$ as above, $Z \cdot \kappa\left(b_{0}\right)=0$, where the action is induced by the adjoint action. (This fact is a simple consequence of vanishing of the Lie derivative $L_{\tilde{\eta}} \kappa$ in the Cartan bundle $B$.) Now decompose $\kappa\left(b_{0}\right)$ according to $\mathfrak{g}=\mathfrak{g}_{-1} \oplus \mathfrak{g}_{0} \oplus \mathfrak{g}_{1}$. The component $\kappa_{-1}\left(b_{0}\right)$ is well known to be harmonic, because it is the lowest homogeneous component, so it represents the harmonic torsion $\tau$. Since the adjoint action is compatible with the gradings, we have $Z \cdot \kappa_{-1}\left(b_{0}\right)=0$. Now $Z$ commutes with $\mathfrak{g}_{1}$, so this condition just means that all values $\tau_{x_{0}}(u, v)$ are in $C(\alpha) \subset T_{x_{0}} M$.

On the other hand, proposition 2.15 gives that for any $\xi \in T(\alpha)$, the function corresponding to $\tau$ must have values in $\mathbb{V}_{s t}(A)$. In particular, by part (3)(f) of lemma 3.4, $\tau\left(x_{0}\right)$, viewed as a skew symmetric bilinear map $\mathbf{R}^{n} \times \mathbf{R}^{n} \rightarrow S^{2} \mathbf{R}^{2} \otimes C(\alpha)$, vanishes whenever one of the arguments is in $W$. Now varying $\xi$ gives different $W$ spanning all of $\mathbf{R}^{n}$, so we conclude that $\tau\left(x_{0}\right)=0$. By part (a) of (2), $C(\alpha)$ in normal coordinates corresponds to higher order fixed points with rank one isotropy, so (3) follows. 
(4) By lemma 3.4, any $\xi \notin \operatorname{ker}(\alpha)$ gives rise to $A$ for which $\mathbb{U}_{s s}(A)=$ $\{0\}$. Thus the statement on $\rho$ follows from (3) above and part (3) of corollary 2.14. If the geometry is torsion free, then all harmonic curvatures vanish on an open neighborhood of $c \backslash\left\{x_{0}\right\}$, so the geometry is flat there.

To prove $(4)(\mathrm{b})$, let $\tilde{c}(s)=\exp \left(b_{0}, s X\right)$ for $X \in T_{\mathfrak{g}_{-}}(Z)$, so $c=\pi \circ \tilde{c}$. Because $\eta$ preserves $c$, it is tangent along $c$. Recall the notation of lemma 3.4: $X \in V^{\circ} \otimes W$ and $\mathfrak{a}(X)=V^{\circ} \otimes \mathbf{R}^{n}+\mathbf{R}^{2} \otimes W$. Let $\tilde{\tau}$ be the $P$-equivariant function on $B$ corresponding to the harmonic torsion. By part (3) immediately above, $\tilde{\tau}\left(b_{0}\right)=0$, so by proposition 2.9, $\tilde{\tau} \in \mathbb{V}_{s s}$ along $\tilde{c}$. This subspace is identified in part (3)(e) of lemma 3.4. The first term, $\mathbb{V}_{s s}^{1} \otimes \mathbb{V}_{s t}^{2}$, corresponds to two-forms, the values of which are endomorphisms that vanish on $V$. The second term, $\mathbb{V}_{s t}^{1} \otimes \mathbb{V}_{s s}^{2}$, corresponds to two-forms, the values of which are endomorphisms with image in $W$. Thus $\tau_{x}(u, v) \in \mathfrak{a}(\eta(x))$ for $x \in c \backslash\left\{x_{0}\right\}$, as claimed.

From the description of $\mathbb{V}_{s s}$, one can also see that $i_{\eta} \tau=0$ and that $\tau_{x}(u, v)$ vanishes when $u, v \in \mathfrak{a}(\eta(x))$. Finally, inserting an element of $V^{\circ}$ into $\mathbb{V}_{s t}^{1}(A)$ gives an element of $V \otimes V^{\circ}$, while inserting an element of $W$ into $\mathbb{V}_{s t}^{2}(A)$ gives an element of $W^{\circ} \otimes W$, and the last remaining claim follows. $\diamond$

3.2. Almost Quaternionic structures. A quaternion-Kähler metric on a smooth manifold has an underlying almost quaternionic structure, which is automatically integrable. These integrable structures are often referred to as quaternionic structures, and the integrability is equivalent to torsion freeness of the associated Cartan geometry. Almost quaternionic structures are very similar to almost Grassmannian structures of type $(2,2 n)$, since the Lie algebras governing the two geometries have the same complexification. As in the Grassmanian case, there is a relation to conformal geometry in the lowest dimensional case $n=1$. This time, however, the corresponding conformal structures are not of split signature but of definite signature. In view of this close analogy, we can carry over most of the results from the Grassmannian case rather easily and treat this geometry quite briefly. 
The homogeneous model is the quaternionic projective space $\mathbf{H P}^{n}$, viewed as a homogeneous space of $P G L(n+1, \mathbf{H})$. The corresponding geometries can be described as first order structures with structure group $G_{0}=S\left(\mathbf{H}^{*} \times G L(n, \mathbf{H})\right)<G L(4 n, \mathbf{R})$, with factors corresponding to scalar multiplications by non-zero quaternions (which are not quaternionically linear maps since $\mathbf{H}$ is non-commutative) and quaternionically linear automorphisms. Such a geometry on a smooth manifold $M^{4 n}$ is given by a rank three subbundle $\mathcal{Q} \subset \operatorname{End}(T M)$, locally spanned by $I, J$, and $I J$ for two anti-commuting almost complex structures $I$ and $J$. See section 4.1 .8 of [4 for more details on these geometries.

The Lie algebra governing the geometry is $\mathfrak{g}=\mathfrak{s l}(n+1, \mathbf{H})$ endowed with a $|1|$-grading $\mathfrak{g}=\mathfrak{g}_{-1} \oplus \mathfrak{g}_{0} \oplus \mathfrak{g}_{1}$ given by blocks of size 1 and $n$ in quaternionic matrices. Thus $\mathfrak{g}_{-1} \cong L_{\mathbf{H}}\left(\mathbf{H}, \mathbf{H}^{n}\right), \mathfrak{g}_{0} \cong \mathfrak{s}(\mathfrak{g l}(1, \mathbf{H}) \oplus$ $\mathfrak{g l}(n, \mathbf{H}))$ and $\mathfrak{g}_{1} \cong L_{\mathbf{H}}\left(\mathbf{H}^{n}, \mathbf{H}\right)$. In particular, there is just one nonzero orbit of $G_{0}$ in $\mathfrak{g}_{1}$, which behaves as in the rank two case for Grassmannian structures. The spaces $\Lambda^{2} \mathfrak{g}_{ \pm 1}$ and $S^{2} \mathfrak{g}_{ \pm 1}$ can be decomposed according to their quaternionic linearity properties. The relevant torsion and curvature components are specified in terms of these subspaces, see section 4.1 .8 of [4]. Now the following results can be proved analogously to lemma 3.2. There is a small simplification in the description of $F_{\mathfrak{g}_{-}}(Z)$, however, coming from the fact that any nonzero $X \in L_{\mathbf{H}}\left(\mathbf{H}, \mathbf{H}^{n}\right)$ is injective.

Lemma 3.6. Let $Z \in \mathfrak{g}_{1} \cong L_{\mathbf{H}}\left(\mathbf{H}^{n}, \mathbf{H}\right)$ be any nonzero element. Then

(1) The subspaces determined by $Z$ are

$$
\begin{gathered}
\{0\}=C_{\mathfrak{g}_{-}}(Z) \subset F_{\mathfrak{g}_{-}}(Z)=\left\{X \in L_{\mathbf{H}}\left(\mathbf{H}, \mathbf{H}^{n}\right): Z X=0\right\} \\
T_{\mathfrak{g}_{-}}(Z)=\left\{X \in L_{\mathbf{H}}\left(\mathbf{H}, \mathbf{H}^{n}\right): Z X=\operatorname{Id}_{\mathbf{H}}\right\}
\end{gathered}
$$

and mapping $X$ to im $(X)$ identifies $T_{\mathfrak{g}_{-}}(Z)$ with the space of all quaternionic lines in $\mathbf{H}^{n}$ having zero intersection with $\operatorname{ker}(Z)$.

(2) For $X \in T_{\mathfrak{g}_{-}}(Z)$, let $A=[Z, X]$ and $W=\operatorname{im}(X) \subset \mathbf{H}^{n}$. Then the eigenvalues of $A$ on $\mathfrak{g}_{-1}$ are all negative. Denoting by $\mathbb{V}$ and $\mathbb{U}$ the representations of $G_{0}$ corresponding to the harmonic torsion and the harmonic curvature, we have $\mathbb{V}_{s s}(A)=\{0\}$ and 


$$
\begin{aligned}
& \mathbb{U}_{s t}(A)=\{0\} . \text { Finally, } \\
& \qquad \mathbb{V}_{s t}(A) \subset \Lambda^{2} \mathfrak{g}_{1} \otimes L_{\mathbf{H}}(\mathbf{H}, W) \subset \Lambda^{2} \mathfrak{g}_{1} \otimes \mathfrak{g}_{-1} .
\end{aligned}
$$

The translation to geometry is also closely similar to the case of Grassmannian structures. The natural structure on $T_{x_{0}} M$ is the three dimensional subspace $\mathcal{Q}_{x_{0}} \subset L\left(T_{x_{0}} M, T_{x_{0}} M\right)$ spanned by two anti-commuting almost complex structures and their product; a tangent vector $\xi \in$ $T_{x_{0}} M$ determines a quaternionic subspace of $T_{x_{0}} M$ generated by $\xi$, which by definition is spanned by $\xi$ and the elements $q(\xi)$ for $q \in \mathcal{Q}_{x_{0}}$. Using this structure, the following result is proved precisely as proposition 3.3 ,

Theorem 3.7. Let $M$ be a smooth manifold of dimension $4 n$ endowed with an almost quaternionic structure $\mathcal{Q} \subset L(T M, T M)$. Let $\eta \in$ $\inf (M)$, vanishing to higher order at $x_{0} \in M$ with isotropy $\alpha \in T_{x_{0}}^{*} M$.

(1) The sets $C(\alpha) \subset F(\alpha) \subset T_{x_{0}} M$ are

$\{0\}=C(\alpha) \subset F(\alpha)=\left\{\xi: \alpha(\xi)=0\right.$ and $\left.\alpha(q(\xi))=0 \forall q \in \mathcal{Q}_{x_{0}}\right\}$

and

$$
T(\alpha)=\left\{\xi: \alpha(\xi)=1 \text { and } \alpha(q(\xi))=0 \quad \forall q \in \mathcal{Q}_{x_{0}}\right\}
$$

(2) In any normal coordinate chart centered at $x_{0}$, there is an open neighborhood $U$ of 0 such that

(a) The higher order fixed point $x_{0}$ is smoothly isolated in the strongly fixed set of the flow, and points of $F(\alpha) \cap U$ are fixed.

(b) On the cone

$$
S=\left\{\xi \in U: \alpha(\xi) \neq 0, \alpha(q(\xi))=0 \forall q \in \mathcal{Q}_{x_{0}}\right\},
$$

the flow in normal coordinates acts by

$$
\varphi_{\eta}^{t}(\xi)=\frac{1}{1+t \alpha(\xi)} \cdot \xi \quad \text { for } t \alpha(\xi)>0
$$

(3) There is an open neighborhood of $S \backslash\{0\}$ on which the geometry is locally flat. 


\subsection{A general result for parabolic contact structures. These} geometries are associated to contact gradings, $|2|$-gradings such that $\mathfrak{g}_{-2}$ has dimension one and the Lie bracket [, ]: $\mathfrak{g}_{-1} \times \mathfrak{g}_{-1} \rightarrow \mathfrak{g}_{-2}$ is a non-degenerate bilinear form. Such gradings can only exist on simple, not on semisimple, Lie algebras; moreover, on each complex simple Lie algebra and most non-compact real forms, there is a unique grading of this type. On the manifold $M$, the subspace $\mathfrak{g}_{-1}$ corresponds to a distribution $T^{-1} M$ of corank one, and the condition on the bracket exactly means that, for a regular parabolic geometry, the distribution is contact. The subalgebra $\mathfrak{g}_{0} \subset L\left(\mathfrak{g}_{-1}, \mathfrak{g}_{-1}\right)$ gives an additional structure on the contact distribution. The best known example of these geometries is partially integrable almost CR structures, for which this additional structure is an almost complex structure on the contact distribution. CR structures will be discussed in more detail in subsection 3.4 below.

For parabolic contact structures, having a higher order fixed point is a weaker condition than for the geometries corresponding to $|1|$-gradings we have treated so far. The difference is that for higher gradings, the subalgebra $\mathfrak{p}_{+}$does not act trivially on the representation $\mathfrak{g} / \mathfrak{p}$, for which $T M$ is the associated bundle. Only the subalgebra $\mathfrak{g}_{2}$ is trivial on $\mathfrak{g} / \mathfrak{p}$, while $\mathfrak{g}_{1}$ actually injects into $L\left(\mathfrak{g}_{-2}, \mathfrak{g}_{-1}\right)$. Hence the fact that an infinitesimal automorphism $\eta$ has a higher order fixed point at $x_{0} \in M$ only implies that the differential $D_{x_{0}} \varphi_{\eta}^{t}: T_{x_{0}} M \rightarrow T_{x_{0}} M$ satisfies $D_{x_{0}} \varphi_{\eta}^{t}(\xi)-\xi \in T_{x_{0}}^{-1} M$ and restricts to the identity map on the contact subspace $T_{x_{0}}^{-1} M$.

In order that $D_{x_{0}} \varphi_{\eta}^{t}=\mathrm{Id}$, the isotropy of $\eta$ must be contained in the subbundle of $T_{x_{0}}^{*} M$ corresponding to the $P$-invariant subspace $\mathfrak{g}_{2} \subset \mathfrak{p}_{+}$, which equals the annihilator of the contact subspace $T_{x_{0}}^{-1} M$. For these isotropies, we can now prove a uniform result for all parabolic contact structures. The algebraic background needed to treat this case is a simple standard result, which at the same time provides the key to the classification of contact gradings:

Lemma 3.8. Let $\mathfrak{g}$ be a real or complex simple Lie algebra endowed with a contact grading $\mathfrak{g}=\mathfrak{g}_{-2} \oplus \cdots \oplus \mathfrak{g}_{2}$. Then for any non-zero 
element $Z \in \mathfrak{g}_{2}$ there exists $X \in \mathfrak{g}_{-2} \cap T_{\mathfrak{g}_{-}}(Z)$ such that $[Z, X]=$ $A$ is the grading element of $\mathfrak{g}$. In particular, for any representation $\mathbb{V}$ corresponding to a harmonic curvature component of the geometry determined by $\mathfrak{g}$, we have $\mathbb{V}_{\text {st }}(A)=\{0\}$.

Proof. For the first part, see the proof of proposition 3.2.4 and section 3.2.10 in [4]. The second part then follows since the curvature of a regular, normal, parabolic geometry is concentrated in positive homogeneities, hence so are all harmonic curvature components.

The transition to geometry is also very simple in this case, thanks to the chains, special distinguished curves defined for any parabolic contact structure; these generalize the well known chains for CR structures, see [19], [20], and section 5.3.7 in [4]. Chains are uniquely determined by their initial direction up to reparametrization and, from any point $x$, chains emanate in each direction transverse to the contact hyperplane. They are defined as distinguished curves of the form $\pi(\exp (b, s X))$ with $X \in \mathfrak{g}_{-2}$ and $b \in B$. For a fixed $b$, there is only one such curve, up to parametrization, but varying $b$ along a fiber in the Cartan bundle yields all chains emanating from $\pi(b)$.

Theorem 3.9. Let $M$ be a smooth manifold endowed with a parabolic contact structure and let $\eta \in \mathfrak{i n f}(M)$. Suppose that $\eta$ has a higher order fixed point at $x_{0} \in M$ such that the isotropy $\alpha \in T_{x_{0}}^{*} M$ vanishes on the contact subbundle $T_{x_{0}}^{-1} M$. Then the higher order fixed point $x_{0}$ is smoothly isolated, and there is an open set $U$ with $x_{0} \in \bar{U}$ on which the geometry is locally flat.

Proof. For all $b \in \pi^{-1}\left(x_{0}\right)$, the element $Z \in \mathfrak{p}_{+}$corresponding to the isotropy $\alpha$ via $b$ lies in $\mathfrak{g}_{2}$. The element $X \in \mathfrak{g}_{-2} \cap T_{\mathfrak{g}_{-}}(Z)$ from lemma 3.8 then gives rise to a distinguished curve $\sigma$, which is a chain. By corollary 2.14 any harmonic curvature component vanishes on a neighborhood $U$ of $\sigma \backslash\left\{x_{0}\right\}$, as desired.

It remains to show the centralizer $C_{\mathfrak{g}_{-}}(Z)$ is trivial. Denote by $B$ the Killing form of $\mathfrak{g}$. Then

$$
\left(Y, Y^{\prime}\right) \mapsto B\left(Z,\left[Y, Y^{\prime}\right]\right)=B\left([Z, Y], Y^{\prime}\right)
$$


is nondegenerate on $\mathfrak{g}_{-1}$. Thus $C_{\mathfrak{g}_{-}}(Z)=0=C(\alpha)$, so $x_{0}$ is smoothly isolated by proposition 2.5 (3).

Remark 3.10. In the proof above, varying $b \in \pi^{-1}\left(x_{0}\right)$ gives vanishing of the harmonic curvature along all chains through $x_{0}$. We expect that these form a dense subset of a neighborhood of $x_{0}$, so that the geometry is flat on a neighborhood of $x_{0}$; however, we are not aware of a general proof of this density of chains.

In the case of $C R$ structures (see section 3.4), it is possible to conclude flatness on a neighborhood of $x_{0}$ thanks to the Fefferman construction (see, for example, [21]). Given $\eta$ as above with $D_{x_{0}} \varphi_{\eta}^{t} \equiv I d$, there is a conformal pseudo-Riemannian structure on a neighborhood $V$ of $x_{0}$ product $S^{1}$ and a lift $\hat{\eta}$ of $\eta$ to a conformal Killing field. The chains $\gamma_{X}$ preserved by $\varphi_{\eta}^{t}$ lift to null geodesics preserved by $\varphi_{\hat{\eta}}^{t}$, and the $S^{1}$-fiber over $x_{0}$ is a pointwise fixed null geodesic. This conformal flow is in fact strongly essential at each point of $\left\{x_{0}\right\} \times S^{1}$; more precisely, it is a null translation (see [3, theorem 4.3] or [10, proposition 3.7]).

By the results cited above, $V \times S^{1}$ is conformally flat on a set of the form

$$
\hat{U}=\bigcup_{x \in \Delta} \mathcal{C}(x)
$$

where $\Delta$ is the fiber over $x_{0}$, and $\mathcal{C}(x)$ is the null cone through $x$. Now it is easy to see that the projection of $\hat{U}$ to $M$ is a neighborhood of $x_{0}$, and that it is CR-flat by the Fefferman correspondence.

3.4. Partially integrable almost CR structures. As mentioned before, these are the most important examples of parabolic contact structures. Let $M$ be a connected manifold of odd dimension, say $2 n+1$, endowed with a contact distribution $H=T^{-1} M \subset T M$. An equivalent formulation of the contact condition is that $H$ is locally equal to the kernel of a one-form $\lambda$ such that $\lambda \wedge\left(\wedge^{n} d \lambda\right)$ is nowhere vanishing. Another equivalent description of the maximal non-integrability of $H$ is that for each point $x \in M$, the skew symmetric bilinear map $\mathcal{L}_{x}$ : $H_{x} \times H_{x} \rightarrow T_{x} M / H_{x}$ induced by the Lie bracket of vector fields is non-degenerate. 
An almost $\mathrm{CR}$ structure on $M$ consists of a contact distribution $H$ as above, together with a complex structure on $H$-that is, a smooth bundle map $J: H \rightarrow H$ such that $J^{2}=J \circ J=-$ Id. To obtain a parabolic contact structure, $J$ has to satisfy the following compatibility condition, called partial integrability: $\mathcal{L}_{x}\left(J_{x}(\xi), J_{x}(\eta)\right)=\mathcal{L}_{x}(\xi, \eta)$ for all $x \in M$ and $\xi, \eta \in H_{x}$. Assuming this property, $\mathcal{L}_{x}$ is the imaginary part of a Hermitian form $\mathcal{L}_{x}^{\mathrm{C}}$ on $H_{x}$, uniquely determined up to scale, with values in $\left(T_{x} M / H_{x}\right) \otimes \mathbf{C}$, which is called the Levi-form at $x$. The signature $(p, q)$, where $p \geq q$, of $\mathcal{L}_{x}^{\mathrm{C}}$ is well-defined and constant on $M$. When the Levi-forms are definite, the almost CR structure is called strictly pseudoconvex. Strictly pseudoconvex almost CR structures are infinitesimally modeled on $S^{2 n+1} \subset \mathrm{C}^{n+1}$.

The main source of almost CR manifolds are real hypersurfaces in complex manifolds. Indeed, if $M \subset N$ is such a hypersurface, for $N$ a complex manifold, then for each $x \in M$, define $H_{x} \subset T_{x} M \subset T_{x} N$ to be the maximal complex subspace contained in $T_{x} M$, so $H_{x}=T_{x} M \cap$ $i T_{x} M$. The spaces $H_{x}$ form a smooth distribution of corank one on $M$, which is generically contact. Then $J$ is the restriction of the complex structure on $T_{x} N$ to $H_{x}$. Integrability of the complex structure on $N$ implies partial integrability of the resulting almost CR structure on $M$; in fact, it satisfies a stronger condition, called integrability, and hence is called a $C R$-structure.

The general formulation of integrability is as follows. For a partially integrable almost CR structure $(M, H, J)$, for any two sections $\xi, \eta \in$ $\Gamma(H)$, the difference $[\xi, \eta]-[J(\xi), J(\eta)]$ lies in $\Gamma(H)$. Now define an analog of the Nijenhuis tensor

$$
\begin{aligned}
N: \quad & \Gamma(H) \times \Gamma(H) \rightarrow \Gamma(H) \\
& (\xi, \eta) \mapsto[\xi, \eta]-[J(\xi), J(\eta)]+J([J(\xi), \eta]+[\xi, J(\eta)])
\end{aligned}
$$

It is bilinear over smooth functions and thus indeed induces a tensor $N \in \Gamma\left(\Lambda^{2} H^{*} \otimes H\right)$. A partially integrable almost CR structure is integrable if $N \equiv 0$. Note that by construction, the CR Nijenhuis tensor $N$ is of type $(0,2)$, conjugate linear in both variables, meaning $N(J(\xi), \eta)=N(\xi, J(\eta))=-J(N(\xi, \eta))$. 
The description of partially integrable almost CR structures of signature $(p, q)$ as parabolic geometries uses the group $G=P S U(p+1, q+1)$ and the parabolic subgroup $P<G$ defined as the stabilizer of a point in the projective space $\mathbf{C} \mathbf{P}^{p+q+1}$ corresponding to an isotropic complex line in $\mathbf{C}^{p+1, q+1}$. Thus the homogeneous space $G / P$ can be identified with the complex projectivization of the light cone in $\mathbf{C}^{p+1, q+1}$. For more details, see section 4.2.4 of the book [4].

The $|2|$-grading on the Lie algebra $\mathfrak{s u}(p+1, q+1)$ determined by $P$ is given by splitting matrices into blocks with respect to a basis starting with a vector $v$ in the isotropic line stabilized by $P$, ending with a complementary isotropic vector $w$, and having an orthonormal basis of $\operatorname{span}\{v, w\}^{\perp}$ in the middle. These matrices have the form

$$
\left.\left\{\begin{array}{ccc}
a & Z & i z \\
X & A-\frac{a-\bar{a}}{p+q} \mathrm{Id} & -\mathbb{I} Z^{*} \\
i x & -X^{*} \mathbb{I} & -\bar{a}
\end{array}\right): \begin{array}{l}
a \in \mathbf{C} \\
x, z \in \mathbf{R} \\
X \in \mathbf{C}^{n}, Z \in \mathbf{C}^{n *} \\
A \in \mathfrak{s u}(p, q)
\end{array}\right\}
$$

where $\mathbb{I}$ is the diagonal matrix $\operatorname{Id}_{p} \oplus\left(-\operatorname{Id}_{q}\right)$. In the grading, $\mathfrak{g}_{-2}$ is the subspace corresponding to $i x, \mathfrak{g}_{-1}$ to $X$, and so on. In particular, $\mathfrak{g}_{-}$and $\mathfrak{p}_{+}$both are complex Heisenberg algebras of signature $(p, q)$, which reflects the CR structure. The group $G_{0}$ is the conformal unitary group of the Hermitian form of signature $(p, q)$ on $\mathfrak{g}_{ \pm 1}$ with the natural extension of the action to $\mathfrak{g}_{ \pm 2} \cong\left[\mathfrak{g}_{ \pm 1}, \mathfrak{g}_{ \pm 1}\right]$, and $P$ is $G_{0} \ltimes \operatorname{Heis}_{p, q}$.

For $Z \in \mathfrak{g}_{1}$ and $X \in \mathfrak{g}_{-1}$, the bracket $[Z, X]=\left(Z X, \mathbb{I} Z^{*} X^{*} \mathbb{I}-X Z\right) \in$ $\mathfrak{g}_{0} \cong \mathbf{C} \oplus \mathfrak{s u}(p, q)$. The further brackets we will want are

$$
\begin{aligned}
{[[Z, X], X] } & =-2 Z X X+X^{*} \mathbb{I} X \mathbb{I} Z^{*} \\
{[[Z, X], Z] } & =2 Z X Z-Z \mathbb{I} Z^{*} X^{*} \mathbb{I}
\end{aligned}
$$

Next we find the decomposition of $\mathfrak{p}_{+}$into $P$-orbits. The nonzero elements of $\mathfrak{g}_{2}$ form one $P$-orbit. Non-degeneracy of the bracket $\mathfrak{g}_{1} \times$ $\mathfrak{g}_{1} \rightarrow \mathfrak{g}_{2}$ implies that any element in $\mathfrak{p}_{+}$with non-zero component in $\mathfrak{g}_{1}$ is conjugate into $\mathfrak{g}_{1}$ by an element of $P$. On $\mathfrak{g}_{1}$, the $P$-action reduces to the standard action of the conformal unitary group, so there are three $P$-orbits of elements not contained in $\mathfrak{g}_{2}$, corresponding to the sign of 
the inner product of the projection of the element to $\mathfrak{p}_{+} / \mathfrak{g}_{2} \cong \mathbf{C}^{p, q}$. The main difference for our results is between null and non-null elements. We will refer to the isotropies at higher order fix points corresponding to these two cases as null, or non-null, transversal isotropy, respectively. The case of isotropy in $\mathfrak{g}_{2}$ was already treated in section 3.3 .

To formulate our results, it remains to describe the harmonic curvature. CR structures have one harmonic torsion and one harmonic curvature. The harmonic torsion is the CR Nijenhuis tensor $N$ defined above, and its vanishing, which by definition is equivalent to integrability, is also equivalent to torsion freeness of the parabolic geometry. The harmonic curvature $\rho$ is a section of $\Lambda^{2} H^{*} \otimes H^{*} \otimes H$; it is a partially defined twoform of type $(1,1)$ with values in skew-Hermitian endomorphisms of the contact subbundle $H$. It is totally trace free and has some additional symmetries, which will not be relevant for our purpose below.

Propositions 3.13 and 3.15 give a fairly detailed description of the flow around a higher order fixed point with non-null or null isotropy, respectively. Together with proposition 3.9 , these imply:

Theorem 3.11. Let $M$ be a manifold with a partially integrable almost $C R$ structure and let $\eta \in \mathfrak{i n f}(M)$ vanish to higher order at $x_{0} \in M$ with isotropy $\alpha \in T_{x_{0}}^{*} M$. Then

(1) The harmonic torsion vanishes at $x_{0}$. If the structure is integrable, then the harmonic curvature vanishes at $x_{0}$.

(2) If $\alpha$ annihilates $H_{x_{0}} \subset T_{x_{0}} M$ or if the projection of $\alpha$ to $H_{x_{0}}^{*}$ is non-null, then there is an open set $U \subset M$ with $x_{0} \in \bar{U}$ on which the restriction of the almost $C R$ structure is flat; in particular, this conclusion holds if $M$ is strictly pseudoconvex.

In the case that the $\mathrm{CR}$ structure on $M$ is real analytic and integrable, Beloshapka [13] and Loboda [14] proved that existence of nontrivial $\eta \in \mathfrak{i n f}(M)$ vanishing to higher order implies that $\mathrm{M}$ is flat. Their proof involves calculations with Taylor series in Moser normal coordinates.

3.4.1. Non-null transverse isotropy. As before, we start by collecting the algebraic background needed to treat this case. 
Lemma 3.12. Let $Z \in \mathfrak{g}_{1}$ be non-isotropic, so $Z \mathbb{I} Z^{*} \neq 0$. Then

(1) The sets associated to $Z$ are

$$
\begin{gathered}
\{0\}=C_{\mathfrak{g}_{-}}(Z) \subset F_{\mathfrak{g}_{-}}(Z)=\left\{X \in \mathfrak{g}_{-1}: Z X=X^{*} \mathbb{I} X=0\right\} \\
T_{\mathfrak{g}_{-}}(Z)=\left\{\frac{2}{Z \mathbb{I} Z^{*}} \mathbb{I} Z^{*}\right\}
\end{gathered}
$$

(2) Let $X_{0}=\frac{2}{Z \mathbb{I} Z^{*}} \mathbb{I} Z^{*}$, and let $A=\left[Z, X_{0}\right]$. Then $A$ belongs to $\mathfrak{g}_{0}$. All eigenvalues of $A$ on $\mathfrak{g}_{-}$are negative, and for any representation $\mathbb{V}$ corresponding to a harmonic curvature or torsion component, $\mathbb{V}_{s t}(A)=0$.

Proof. For any non-zero $i x \in \mathfrak{g}_{-2}$ the map $W \mapsto[i x, W]$ is an injection $\mathfrak{g}_{1} \rightarrow \mathfrak{g}_{-1}$. Because $Z \in \mathfrak{g}_{1}$, the sets $F_{\mathfrak{g}_{-}}(Z)$ and $C_{\mathfrak{g}_{-}}(Z)$ are in $\mathfrak{g}_{-1}$. Now let $X \in \mathfrak{g}_{-1}$. If $[Z, X]=0$, then $Z X=0$. From $[[Z, X], X]=0$, it follows that $\mathbb{I} Z^{*}$ and $X$ are linearly dependent. But if $X=\lambda \mathbb{I} Z^{*}$, then $Z X=\lambda Z \mathbb{I} Z^{*}$. Since $Z$ is non-isotropic, $\lambda=0$, so $C_{\mathfrak{g}_{-}}(Z)=0$.

For $X \in \mathfrak{g}_{-1}$, grading considerations imply $X \in F_{\mathfrak{g}_{-}}(Z)$ if and only if $\operatorname{ad}(X)^{2}(Z)=0$. If $X$ and $\mathbb{I} Z^{*}$ are linearly independent, then $[[Z, X], X]$ vanishes if and only if $Z X=\langle X, X\rangle=0$. If, on the other hand, $X=\lambda \mathbb{I} Z^{*}$ for $\lambda \in \mathbb{C}$, vanishing of $[[Z, X], X]$ is equivalent to $|\lambda|^{2}=2 \lambda^{2}$, which is satisfied only for $\lambda=0$. The description of $F_{\mathfrak{g}_{-}}(Z)$ now follows. Now suppose $X \in T_{\mathfrak{g}_{-}}(Z)$. Then $[[Z, X], Z]=2 Z$ implies $X^{*} \mathbb{I}=\lambda Z$, and

$$
2 \lambda Z \mathbb{I} Z^{*}-\lambda Z \mathbb{I} Z^{*}=2 Z X-\lambda Z \mathbb{I} Z^{*}=2
$$

The only possibility is thus $\lambda=\frac{2}{Z \mathbb{I} Z^{*}}$. It is easy to verify that for $X_{0}=\frac{2}{Z \mathbb{I} Z^{*}} \mathbb{I} Z^{*}$, we have $\left[\left[Z, X_{0}\right], X_{0}\right]=-2 X_{0}$, so $T_{\mathfrak{g}_{-}}(Z)=\left\{X_{0}\right\}$, as claimed.

For (2), compute $Z X_{0}=2$ and $\mathbb{I} Z^{*} X_{0}^{*} \mathbb{I}-X_{0} Z=0$, whence $A=\left[Z, X_{0}\right]$ is the block diagonal matrix with entries $(2,0, \ldots, 0,-2)$, which is twice the grading element. The statement on $\mathbb{V}_{s t}(A)$ then immediately follows from homogeneity considerations as in the proof of lemma 3.8

Here is the resulting proposition for non-null transverse isotropy.

Proposition 3.13. Let $M$ be a manifold endowed with a partially integrable almost $C R$ structure and let $\eta \in \mathfrak{i n f}(M)$ vanish to higher order 
at $x_{0} \in M$. Assume further that the isotropy $\alpha \in T_{x_{0}}^{*} M$ of $\eta$ restricts to a non-null element of $\left(H_{x_{0}}\right)^{*}$. Then

(1) The sets $C(\alpha) \subset F(\alpha)$ in $T_{x_{0}} M$ are

$$
\{0\}=C(\alpha) \subset F(\alpha)=\left\{\xi \in H_{x_{0}}: \mathcal{L}_{x_{0}}(\xi, J(\xi))=0, \alpha(\xi)=0\right\}
$$

The real line spanned by $T(\alpha)$ consists of all elements dual to real multiples of $\left.\alpha\right|_{H_{x_{0}}}$.

(2) Let $b_{0} \in \pi^{-1}\left(x_{0}\right)$ be a point such that $\alpha$ corresponds via $b_{0}$ to an element of $\mathfrak{g}_{1}$. Then in the normal coordinate chart determined by $b_{0}$, there is an open neighborhood $U$ of 0 such that:

(a) The higher order fixed point $x_{0}$ is smoothly isolated in the strongly fixed set. Elements of $U \cap F(\alpha)$ are zeros of $\eta$.

(b) Let

$$
\ell=\left\{\xi \in H_{x_{0}}: \operatorname{ker}\left(\mathcal{L}_{x_{0}}(\xi, J(\cdot))\right)=\operatorname{ker}(\alpha) \cap H_{x_{0}}\right\}
$$

Then $\ell$ is a line, and on $\ell \cap U$, the flow acts by $\xi \mapsto \frac{1}{1+t \alpha(\xi)} \xi$ whenever $\operatorname{t} \alpha(\xi)>0$.

(3) There is an open neighborhood of $\ell \backslash\{0\}$ in $U$ on which the geometry is locally flat.

Proof. We fix $b_{0}$ as in (2) and let $Z \in \mathfrak{g}_{1}$ be the element corresponding to $\alpha$ via $b_{0}$. Then $C_{\mathfrak{g}_{-}}(Z)=\{0\}$ by lemma 3.12 and as in the proof of proposition 2.16, this implies that $C(\alpha)=0$. Next, lemma 3.12 implies the description of $F(\alpha)$, and it shows that $T(\alpha)$ consists of the unique element $\xi_{0} \in \ell$ such that $\alpha\left(\xi_{0}\right)=2$, so (1) is proved. Then (2) follows immediately from propositions 2.5, 2.12 (with analogous simplifications as in proposition 2.17) and 2.8.

3.4.2. Null transverse isotropy. In this case, we cannot deduce a local flatness result, so we will focus on proving vanishing of the curvature on a complex curve of higher order fixed points through $x_{0}$ and providing the algebraic background for a more detailed analysis. It is possible to deduce restrictions on torsion and curvature outside of the strongly fixed set as was done for almost Grassmannian structures in part (4) of proposition 3.5. The CR statements are rather complicated to formulate, so we do not prove them in detail here. 
Lemma 3.14. Let $Z \in \mathfrak{g}_{1}$ be isotropic, so $Z \mathbb{I} Z^{*}=0$. Then

(1) The sets associated to $Z$ are

$$
\begin{gathered}
\mathbf{C} \cdot \mathbb{I} Z^{*}=C_{\mathfrak{g}_{-}}(Z) \subset F_{\mathfrak{g}_{-}}(Z)=\left\{X \in \mathfrak{g}_{-1}: Z X=X^{*} \mathbb{I} X=0\right\} \\
T_{\mathfrak{g}_{-}}(Z)=\left\{X \in \mathfrak{g}_{-1}: Z X=1, X^{*} \mathbb{I} X=0\right\}
\end{gathered}
$$

(2) For $X \in T_{\mathfrak{g}_{-}}(Z)$, let $A=[Z, X] \in \mathfrak{g}_{0}$. Then $A$ has nonpositive eigenvalues on $\mathfrak{g}_{-}$, and the 0 -eigenspace equals $C_{\mathfrak{g}_{-}}(Z)$. For $\mathbb{V} \subset \Lambda^{(0,2)} \mathfrak{g}_{1} \otimes \mathfrak{g}_{-1}$ the representation corresponding to the harmonic torsion, $\mathbb{V}_{s t}(A) \subset \Lambda^{2} \mathfrak{g}_{1} \otimes X^{\perp}$, and

$$
\mathbb{V}_{s s}(A) \subset\left(\mathbf{C} \cdot \mathbb{I} X^{*}\right) \wedge\left(\operatorname{ker}(X) \cap Z^{\perp}\right) \otimes \mathbf{C} X
$$

Let $\mathbb{U} \subset \Lambda^{(1,1)} \mathfrak{g}_{1} \otimes \mathfrak{g}_{0}$ be the representation corresponding to the harmonic curvature, and let $\Phi \in \mathbb{U}_{s t}(A)$. If

$$
\Phi\left(Y_{1}, Y_{2}\right) \cdot Z=0 \quad \forall Y_{1}, Y_{2} \in \mathfrak{g}_{-1}
$$

then $\Phi\left(Y_{1}, Y_{2}\right)=0$ whenever $Y_{1} \in \mathbf{C} X$ and $Y_{2} \in \mathfrak{g}_{-1}$.

Proof. As in the proof of lemma 3.12, $F_{\mathfrak{g}_{-}}(Z) \subset \mathfrak{g}_{-1}$, and $X \in \mathfrak{g}_{-1}$ lies in $F_{\mathfrak{g}-}(Z)$ if and only if $\operatorname{ad}(X)^{2}(Z)=0$. As before, $[Z, X]=0$ implies $X=\lambda \mathbb{I} Z^{*}$ for some $\lambda \in \mathbf{C}$. Conversely, any complex multiple of $\mathbb{I} Z^{*}$ commutes with $Z$, so $C_{\mathfrak{g}_{-}}(Z)$ is as claimed in (1). Next suppose $[[Z, X], X]=0$. Then $X$ and $\mathbb{I} Z$ are linearly dependent, in which case $X$ is in $C_{\mathfrak{g}_{-}}(Z)$, or $Z X=0=X^{*} \mathbb{I} X$. The description of $F_{\mathfrak{g}_{-}}(Z)$ is now proved.

For $X \in T_{\mathfrak{g}_{-}}(Z)$, grading considerations imply that $X \in \mathfrak{g}_{-1}$, and then $X$ is linearly independent from $\mathbb{I} Z^{*}$, since otherwise $[Z, X]=0$. But then $[[Z, X], X]=-2 X$ is equivalent to $Z X=1$ and $X^{*} \mathbb{I} X=0$. These two conditions also imply that $[[Z, X], Z]=2 Z$, so the description of $T_{\mathfrak{g}_{-}}(Z)$ follows.

Now, for $X \in T_{\mathfrak{g}_{-}}(Z)$, let $A=[Z, X]$ as in (2). The $A$-action on $\mathfrak{g}_{-2}$ is multiplication by $-2 Z X=-2$, while the action on $\mathfrak{g}_{-1}$ maps $Y$ to $-Y-Z Y X+X^{*} \mathbb{I} Y \mathbb{I} Z^{*}$. Then $X \in \mathfrak{g}_{-1}$ spans the -2 -eigenspace of $A$ in $\mathfrak{g}_{-1} ; \operatorname{ker}(Z) \cap X^{\perp}$ is the -1 -eigenspace; and $\mathbb{I} Z^{*}$ spans the 0 -eigenspace. Thus all eigenvalues of $A$ on $\mathfrak{g}_{-}$are nonpositive, and the 0-eigenspace is $C_{\mathfrak{g}_{-}}(Z)$, as stated in (2). 
The eigenspace structure on $\mathfrak{p}_{+}$follows readily by dualization:

\begin{tabular}{|c|c|}
\hline subspace & eigenvalue \\
\hline $\mathbf{C} \cdot \mathbb{I} X^{*}$ & 0 \\
$\operatorname{ker}(X) \cap Z^{\perp}$ & 1 \\
$\mathfrak{g}_{2} \oplus \mathbf{C} Z$ & 2 \\
\hline
\end{tabular}

Now we can analyze the eigenspace structure on $\mathbb{V}$ and $\mathbb{U}$. On $\Lambda^{(0,2)} \mathfrak{g}_{1}$, the eigenvalues of $A$ range from 1 to 3 . (The eigenspaces with eigenvalues 0 and 4 which are present in $\Lambda^{2} \mathfrak{g}_{1}$ both come from the alternating square of a complex line, so they both are contained in $\Lambda^{(1,1)} \mathfrak{g}_{1}$.) Then the values of a map in $\mathbb{V}_{s t}(A)$ lie in the sum of the eigenspaces in $\mathfrak{g}_{-1}$ with negative eigenvalues. This sum is exactly $X^{\perp} \subset \mathfrak{g}_{-1}$, which proves the claim on $\mathbb{V}_{s t}(A)$.

On the other hand, $\mathbb{V}_{s s}(A)$ evidently coincides with the $(-1)$-eigenspace of $A$ in $\mathbb{V}$, which is the space claimed in the proposition.

The eigenvalues of $A$ on $\mathfrak{g}_{0} \subset \mathfrak{g}_{1} \otimes \mathfrak{g}_{-1}$ range from -2 to 2 , so the possible eigenvalues on $\mathbb{U}$ range from -2 to 6 . Since $Z$ spans the $(+2)-$ eigenspace in $\mathfrak{g}_{1}$, the condition that $\Phi\left(Y_{1}, Y_{2}\right) \cdot Z=0$ implies $\Phi\left(Y_{1}, Y_{2}\right)$ is in the sum of $A$-eigenspaces of $\mathfrak{g}_{0}$ with eigenvalues $\geq-1$. If this holds for all $Y_{1}, Y_{2} \in \mathfrak{g}_{-1}$, and if $\Phi \in \mathbb{U}_{s t}(A)$, then the $\Lambda^{(1,1)} \mathfrak{g}_{1}$-components of $\Phi$ lie in the sum of 0 and 1-eigenspaces. This sum in turn is made up of components from the 0 and 1-eigenspaces in $\mathfrak{g}_{1}$, which form the annihilator of $X$. Now the last claim of (2) follows.

Proposition 3.15. Let $M$ be a manifold endowed with a nondegenerate, partially integrable almost $C R$ structure, and let $\eta \in \mathfrak{i n f}(M)$ vanish to higher order at $x_{0} \in M$. Assume further that the isotropy $\alpha \in T_{x_{0}}^{*} M$ of $\eta$ restricts to a nonzero, null element of $\left(H_{x_{0}}\right)^{*}$.

(1) The set $C(\alpha)$ is the complex line spanned by the elements dual to $\left.\alpha\right|_{H_{x_{0}}}$, while

$$
F(\alpha)=\left\{\xi \in H_{x_{0}}: \alpha(\xi)=0, \mathcal{L}(\xi, J \xi)=0\right\}
$$

and

$$
T(\alpha)=\left\{\xi \in H_{x_{0}}: \alpha(\xi)=1, \mathcal{L}(\xi, J \xi)=0\right\}
$$


(2) Let $b_{0} \in \pi^{-1}\left(x_{0}\right)$ be a point such that $\alpha$ corresponds via $b_{0}$ to an element of $\mathfrak{g}_{1}$. Then in the normal coordinate chart determined by $b_{0}$, there is an open neighborhood $U$ of 0 such that

(a) Points of $C(\alpha) \cap U$ are higher order fixed points with transverse null isotropy. Any $\xi \in F(\alpha) \cap U$ is a zero of $\eta$.

(b) On the cone

$$
S=\left\{\xi \in H_{x_{0}}: \alpha(\xi) \neq 0, \mathcal{L}(\xi, J \xi)=0\right\}
$$

the action of the flow along $\eta$ in normal coordinates is

$$
\varphi_{\eta}^{t}(\xi)=\frac{1}{1+t \alpha(\xi)} \cdot \xi \text { for } t \alpha(\xi)>0
$$

(3) The Nijenhuis tensor vanishes on the complex curve of higher order fixed points $C(\alpha) \cap U$ in (1). If the structure is integrable, that is, CR, then the harmonic curvature also vanishes along the complex curve $C(\alpha) \cap U$.

Proof: The descriptions of $C(\alpha), F(\alpha)$ and $T(\alpha)$ in (1) follow immediately from lemma 3.14 and then (2)(a) and (b) follow immediately from propositions 2.5 and 2.12 .

For (3), let $b_{0} \in \pi^{-1}\left(x_{0}\right)$ be such that $Z \in \mathfrak{g}_{1}$ is the element corresponding to $\alpha$ via $b_{0}$. Choose $X \in T_{\mathfrak{g}_{-}}(Z)$ and let $A=[Z, X] \in \mathfrak{g}_{0}$. Then by proposition 2.15 , the functions corresponding to the harmonic torsion and the harmonic curvature must map $b_{0}$ to $\mathbb{V}_{s t}(A)$ and to $\mathbb{U}_{s t}(A)$, respectively. Now from lemma $3.14, \mathbb{V}_{s t}(A)$ consists of maps having values in $X^{\perp}$. But for a fixed $Z$, elements $X \in T_{\mathfrak{g}_{-}}(Z)$ span $\mathfrak{g}_{-1}$ over C. Thus the harmonic torsion vanishes at $x_{0}$. This torsion is a scalar multiple of the Nijenhuis tensor, which then also vanishes.

To complete the argument in the torsion free case, we proceed as in the proof of proposition 3.5. Denote by $\kappa: B \rightarrow \Lambda^{2} \mathfrak{g}_{-}^{*} \otimes \mathfrak{g}$ the Cartan curvature function. Again from section 2.3 of [5], $\left[\kappa_{b_{0}}\left(Y_{1}, Y_{2}\right), Z\right]=0$ for all $Y_{1}, Y_{2} \in \mathfrak{g}_{-}$. If the structure is CR, then the Cartan connection is torsion free; the value $\kappa_{b_{0}}$ is homogeneous of degrees $\geq 2$, and the lowest non-zero homogeneous component is harmonic. Hence taking $Y_{1}, Y_{2} \in \mathfrak{g}_{-1}$, the component in $\mathfrak{g}_{1}$ of $\left[\kappa_{b_{0}}\left(Y_{1}, Y_{2}\right), Z\right]$ coincides with the value of $\left[\rho_{b_{0}}\left(Y_{1}, Y_{2}\right), Z\right]$, where $\rho$ is the function corresponding to 
the harmonic curvature. By lemma 3.14, $\left[\rho_{b_{0}}\left(Y_{1}, Y_{2}\right), Z\right]=0$ together with $\rho\left(b_{0}\right) \in \mathbb{U}_{s t}(A)$ implies $\rho_{b_{0}}\left(Y_{1}, Y_{2}\right)=0$ for $Y_{1} \in \mathbf{C} X$ and $Y_{2} \in$ $\mathfrak{g}_{-1}$. Again, because $T_{\mathfrak{g}_{-}}(Z)$ spans $\mathfrak{g}_{-1}$ over $\mathbf{C}$, the harmonic curvature vanishes at $x_{0}$, and along the strongly fixed component of $x_{0} . \diamond$

Last, we can easily deduce the following local version of the SchoenWebster theorem [22], 23], which generalizes the result of [2] from the conformal Riemannian setting. Versions of this result were obtained by Vitushkin under the additional assumption of integrability in [11] and by Kruzhilin for smooth, integrable CR structures in [12].

Theorem 3.16. Let $M^{2 n+1}$ be a connected, real-analytic, partially integrable, strictly pseudoconvex $C R$ manifold, and let $\eta \in \mathfrak{i n f}(M)$ vanish at $x_{0} \in M$. Then

- There is a neighborhood $U$ of $x_{0}$ invariant by the flow, on which $\left\{\varphi_{\eta}^{t}\right\}$ is bounded and linearizable; or

- $M$ is spherical-that is, locally flat as a CR structure.

Proof: Pick $b_{0} \in \pi^{-1}\left(x_{0}\right)$, and let $Z \in \mathfrak{p}$ be the isotropy of $\eta$ at $x_{0}$ with respect to $b_{0}$. Because $M$ is real-analytic, the full isotropy subalgebra at $x_{0}$ is algebraic; more precisely, the isotropies with respect to $b_{0}$ of all local Killing fields vanishing at $x_{0}$ form an algebraic subalgebra of $\mathfrak{p}$. This fact is a consequence of the so-called Frobenius Theorem of [24, sec 3.4] and [25, thm 3.11]. An algebraic subalgebra is closed under Jordan decomposition (see [26, 4.3.4]), so we may assume $Z$ is either nilpotent or semisimple.

Recall $\mathfrak{p} \cong(\mathbf{C} \times \mathfrak{s u}(n)) \ltimes \mathfrak{u}$, where $\mathfrak{u}$ is the nilpotent radical, isomorphic to a $(2 n+1)$-dimensional Heisenberg Lie algebra. If $Z \in \mathfrak{u}$, then $M$ is flat on a nonempty open set by theorem 3.11, and so $M$ is flat everywhere because it is analytic.

Now suppose $Z$ is semisimple, so it is conjugate into $\mathfrak{g}_{0} \cong \mathbf{C} \times \mathfrak{s u}(n)$. If $Z$ lies in a maximal compact subalgebra of $\mathfrak{g}_{0}$, then $\operatorname{ad}(Z)$ preserves the subspace $\mathfrak{g}_{-}$and a positive-definite inner product on it. This inner product descends to $T_{x_{0}} M$, where it is invariant by $\left\{D_{x_{0}} \varphi_{\eta}^{t}\right\}$, the differential of the flow, and it pulls back via the normal coordinate chart 
determined by $b_{0}$ to a Riemannian metric on a precompact neighborhood $U$ of $x_{0}$, which is invariant by $\left\{\varphi_{\eta}^{t}\right\}$. It follows that the flow is bounded and linearizable on $U$.

Now suppose that $Z$ generates an unbounded 1-parameter subgroup, so it can be written $Z=A+K$, where $A$ is a nonzero multiple of the grading element, and $K \in \mathfrak{s u}(1) \times \mathfrak{s u}(n)$. First compute that for $X \in \mathfrak{g}_{-}$,

$$
e^{t Z} e^{s X}=e^{s a^{t} k_{t}(X)} e^{t Z}
$$

where $\operatorname{Ad}\left(e^{t Z}\right)=\operatorname{Ad}\left(e^{t A}\right) \circ \operatorname{Ad}\left(e^{t K}\right)=a^{t} \cdot k_{t}$, for $a \neq 0$ and $k_{t} \in$ $\mathrm{SU}(1) \times \mathrm{SU}(n)$. We can assume that $a<1$, by replacing $\eta$ with $-\eta$ if necessary. Now proposition 2.1 impies that all the distinguished curves emanating from $x_{0}$ tend to $x_{0}$ under the flow by $\varphi_{\eta}^{t}$, and that $e^{t Z}$ is again a holonomy path with attractor $b_{0}$ along the curves $\exp _{b_{0}}(s X)$. Now $e^{t Z}$ satisfies the hypotheses of proposition 2.8 for all $Y \in \mathfrak{g}_{-}$, so it is also a holonomy path at points projecting onto a neighborhood of $\exp _{b_{0}}(s X)$. The action of $e^{t Z}$ on the representations for the harmonic curvature and torsion is expanding - that is, any vector $w$ in one of these representations satisfies $\left\|e^{t Z}(w)\right\| \rightarrow \infty$ as $t \rightarrow \infty$. By arguments similar to those in proposition 2.9, both the torsion and curvature vanish wherever we have $e^{t Z}$ as a holonomy path. Thus the curvature vanishes on an open set, so it vanishes everywhere by analyticity. $\diamond$

\section{REFERENCES}

[1] T. Nagano and T. Ochiai, "On compact Riemannian manifolds admitting essential projective transformations," J. Fac. Sci. Univ. Tokyo Sect. IA Math., vol. 33, no. 2, pp. 233-246, 1986.

[2] C. Frances, "Local dynamics of conformal vector fields," Geom. Ded., vol. 158, no. 1, pp. 35-59, 2012.

[3] C. Frances and K. Melnick, "Formes normales pour les champs conformes pseudo-riemanniens," Bulletin de la Société Mathématique de France, vol. 141, no. 3, pp. 377-421, 2013.

[4] A. Čap and J. Slovák, Parabolic geometries I, vol. 154 of Mathematical Surveys and Monographs. Providence, RI: American Mathematical Society, 2009.

[5] A. Cap, "Automorphism groups of parabolic geometries," Rend. Circ. Mat. Palermo (2) Suppl., no. 75, pp. 233-239, 2005. 
[6] A. Čap, "Infinitesimal automorphisms and deformations of parabolic geometries," J. Eur. Math. Soc., vol. 10, no. 2, pp. 415-437, 2008.

[7] È. B. Vinberg, "The Weyl group of a graded Lie algebra," Izv. Akad. Nauk SSSR Ser. Mat., vol. 40, no. 3, pp. 488-526, 709, 1976.

[8] J. Alt, "Essential parabolic structures and their infinitesimal automorphisms," SIGMA: Symmetry Integrability Geom. Methods Appl., vol. 7, 2011. Paper 039.

[9] C. Frances and K. Melnick, "Nilpotent groups of conformal flows on compact pseudo-Riemannian manifolds," Duke Math. J., vol. 153, no. 3, pp. 511-550, 2010.

[10] A. Čap and K. Melnick, "Essential killing fields of parabolic geometries: projective and conformal structures," Central European Journal of Mathematics, vol. 11, no. 12, pp. 2053-2061, 2013.

[11] A. G. Vitushkin, "Holomorphic extension of mappings of compact hypersurfaces," Izv. Akad. Nauk SSSR Ser. Mat., vol. 20, pp. 27-33, 1983.

[12] N. G. Kruzhilin, "Local automorphisms and mappings of smooth strictly pseudoconvex hypersurfaces," Math. Ussr Izvestiya, vol. 26, no. 3, pp. 531-552, 1986.

[13] V. K. Beloshapka, "On the dimension of the group of automorphisms of an analytic hypersurface," Izv. Akad. Nauk. SSSR Ser. Math., vol. 14, pp. 223$245,1980$.

[14] A. V. Loboda, "On local automorphisms real-analytic hypersurface," Izv. Akad. Nauk. SSSR Ser. Math., vol. 18, pp. 537-559, 1982.

[15] A. Čap, A. R. Gover, and M. Hammerl, "Holonomy reductions of Cartan geometries and curved orbit decompositions," Duke Math. J., vol. 163, no. 5, pp. 1035-1070, 2014.

[16] C. Frances, "Sur le groupe d'automorphismes des géométries paraboliques de rang 1," Ann. Sci. École Norm. Sup. (4), vol. 40, no. 5, pp. 741-764, 2007.

[17] U. Bader, C. Frances, and K. Melnick, "An embedding theorem for automorphism groups of Cartan geometries," Geom. Funct. Anal., vol. 19, no. 2, pp. 333-355, 2009.

[18] C. Frances, "Dégénerescence locale des transformations conformes pseudoriemanniennes," Ann. Inst. Fourier, vol. 62, pp. 1627-1669, 2012.

[19] A. Čap, J. Slovák, and V. Žádník, "On distinguished curves in parabolic geometries," Transform. Groups, vol. 9, no. 2, pp. 143-166, 2004.

[20] A. Čap and V. Žádník, "On the geometry of chains," J. Differential Geom., vol. 82, no. 1, pp. 1-33, 2009.

[21] L. K. Koch, "Chains, null-chains, and CR geometry," Trans. Amer. Math. Soc., vol. 338, no. 1, pp. 245-261, 1993.

[22] S. Webster, "On the transformation group of a real hypersurface," Trans.Amer. Math. Soc., vol. 231, no. 1, pp. 179-190, 1977. 
[23] R. Schoen, "On the conformal and CR automorphism groups," Geom. Funct. Anal., vol. 5, no. 2, pp. 464-481, 1995.

[24] M. Gromov, "Rigid transformations groups," in Géométrie Différentielle (Paris, 1986) (D. Bernard and Y. Choquet-Bruhat, eds.), pp. 65-139, Paris: Hermann, 1988.

[25] K. Melnick, "A Frobenius theorem for Cartan geometries, with applications," L'Enseignement Mathématique Sér. II, vol. 57, no. 1-2, pp. 57-89, 2011.

[26] D. W. Morris, Ratner's theorem on unipotent flows. Chicago Lectures in Mathematics, University of Chicago Press, 2005.

Andreas Čap

Faculty of Mathematics

University of Vienna

Oskar-Morgenstern-Platz 1

1090 Vienna, Austria

andreas.cap@univie.ac.at
Karin Melnick

Department of Mathematics

University of Maryland

College Park, MD 20742

USA

karin@math.umd.edu 